Budker INP 2003-13

DESY 03-025

\title{
RADIATIVE CORRECTIONS TO QCD AMPLITUDES IN QUASI-MULTI-REGGE KINEMATICS *
}

\author{
V.S. Fadin ${ }^{\dagger}$, M.G. Kozlov ${ }^{\ddagger}$ A.V. Reznichenko ${ }^{\dagger \dagger}$ \\ Institute for Nuclear Physics, 630090 Novosibirsk, Russia \\ and Novosibirsk State University, 630090 Novosibirsk, Russia
}

\begin{abstract}
Radiative corrections to QCD amplitudes in the quasi-multi-Regge kinematics are interesting in particular since the Reggeized form of these amplitudes is used in the derivation of the NLO BFKL. This form is a hypothesis which must be at least carefully checked, if not proved. We calculate the radiative corrections in the one-loop approximation using the $s$-channel unitarity. Compatibility of the Reggeized form of the amplitudes with the $s$-channel unitarity requires fulfillment of the set of nonlinear equations for the Reggeon vertices. We show that these equations are satisfied.
\end{abstract}

${ }^{*}$ Work supported in part by INTAS and in part by the Russian Fund of Basic Researches.

$\dagger$ e-mail address: FADIN@INP.NSK.SU

$\ddagger$ e-mail address: M.G.KOZLOV@INP.NSK.SU

${ }^{\dagger \dagger}$ e-mail address: A.V.REZNICHENKO@INP.NSK.SU 


\section{Introduction}

In the limit of large center of mass energy $\sqrt{s}$ and fixed momentum transfer $\sqrt{-t}$ (Regge limit) the most appropriate approach for the description of scattering amplitudes is given by the theory of complex angular momenta (Gribov-Regge theory). One of remarkable properties of QCD is the Reggeization of its elementary particles. Contrary to QED, where the electron does Reggeize in perturbation theory [1, but the photon remains elementary [2], in QCD the gluon does Reggeize [3]-[5] as well as the quark [6]-[8].

The phenomenon of the Reggeization is very important for high energy QCD. In particular, the BFKL approach [5] to the description of high energy QCD processes is based on the gluon Reggeization. It was assumed in this approach that the amplitudes with colour octets and negative signatures in channels with fixed (not increasing with $s$ ) transferred momenta have the Reggeized form. In the leading logarithmic approximation (LLA), when only the leading terms $\left(\alpha_{S} \ln s\right)^{n}$ are resummed [5], the assumption was made about the amplitudes in the multi-Regge kinematics (MRK). Remind that the MRK means large invariant masses of any pair of final state particles and fixed transverse momenta; we include here the Regge kinematics (RK) in the MRK as a particular case. The Reggeized form of these amplitudes in the LLA was proved [9], so that in this approximation the BFKL approach is completely justified.

Now the BFKL approach is developed in the next-to-leading approximation (NLA), when the terms $\alpha_{S}\left(\alpha_{S} \ln s\right)^{n}$ are also resummed. The kernel of the BFKL equation for the forward scattering ( $t=0$ and colour singlet in the $t$-channel) in the next-to-leading order (NLO) is found [10, 11]. The calculation of the NLO kernel for the non-forward scattering [12] is not far from completion (see [13, [14]). The impact factors of gluons [15] and quarks [16] are calculated in the NLO and the impact factors of the physical (colour singlet) particles are under investigation [17, [18, [19], 20], 21].

The NLO results are obtained assuming the Reggeized form both for the amplitudes in the quasi-multi-Regge kinematics (QMRK), where a pair of produced particles has fixed invariant mass, and for the MRK amplitudes in the NLA. It's clear that these assumptions must be at least carefully checked, if not proved. It can be done by revision of the "bootstrap" relations [12], appearing from the requirement of compatibility of the Reggeized form of the amplitudes with the $s$-channel unitarity. For the elastic amplitudes these relations impose the bootstrap conditions on the colour-octet impact factors and the BFKL kernel in the NLO [12. The conditions for the impact factors of gluons [15] and quarks [16], as well as for the quark part of the kernel [13], were shown to be satisfied at arbitrary space-time dimension $D$. For the gluon part of the kernel fulfillment of the bootstrap condition was proved at $D \rightarrow 4$ [22], in particular, because this part was available at that time only in such limit. Now it can be done at arbitrary $D$, since the kernel at arbitrary $D$ is calculated [23].

Evidently, the bootstrap relations must be satisfied for all amplitudes which were assumed to have the Reggeized form, so that there is an infinite set of such relations. Since the amplitudes are expressed in terms of the gluon trajectory and a finite number of the Reggeon vertices, it is extremely nontrivial to satisfy all these relations. Nevertheless, it occurs 
that all of them can be fulfilled if the vertices and trajectory submit to several bootstrap conditions [24. On the other hand, the fulfillment of all bootstrap relations secures the Reggeized form of the radiative corrections order by order in the perturbation theory. On this way the proof of the Reggeization was constructed in the LLA [9]. An analogous proof can be constructed in the next-to-leading approximation (NLA) as well [24].

The bootstrap relations for the multi-particle production amplitudes give [24, in particular, stronger restrictions on the octet impact factors and kernel, than the relations for the elastic amplitudes. These restrictions are known as the strong bootstrap conditions suggested, without derivation, in [25, 26], which lead to remarkable properties of the colouroctet impact factors and the Reggeon vertices [27, that their ratio is a process-independent function. In the NLO this quite nontrivial property was verified by comparison of such ratio for quarks and gluons [27. Moreover, the process-independent function mentioned above must be the eigenfunction of the octet kernel. In the part concerning the quark contribution to the kernel it is proved rather easily [13, [26], 28]. To do this for the gluon contribution requires much more efforts, but recently it was also done [29].

In this paper we investigate the bootstrap relations for the production amplitudes in the QMRK. We calculate the one-loop radiative corrections to these amplitudes using the $s$-channel unitarity, derive the bootstrap conditions for the production vertices and demonstrate that they are fulfilled.

The next Section contains all necessary definitions and denotations. Then, in Section 3 , we consider the amplitudes with a couple of particles in the fragmentation region of one of colliding particles. We calculate the one-loop radiative corrections for these amplitudes and derive the bootstrap conditions for the Reggeon vertices in the QMRK in Subsection 3.1. In Subsections 3.2, 3.3 and 3.4 we demonstrate that these conditions are satisfied for quark-antiquark, gluon-gluon and quark-gluon production respectively. Next we consider production of a couple of particles with fixed invariant mass in the central region of rapidities. Subsection 4.1 contains the calculation of the one-loop radiative corrections and derivation of the bootstrap conditions. Fulfillment of these conditions is proved in Subsections 4.2 and 4.3 for quark-antiquark and gluon-gluon production respectively. Significance of the obtained results is discussed in Section 5.

\section{Definitions and denotations}

Considering collisions of high energy particles $A$ and $B$ with momenta $p_{A}$ and $p_{B}$ and masses $m_{A}$ and $m_{B}$ we introduce light cone 4 -vectors $p_{1}$ and $p_{2}$ so that

$$
p_{A}=p_{1}+\left(m_{A}^{2} / s\right) p_{2}, p_{B}=p_{2}+\left(m_{B}^{2} / s\right) p_{1}, s=2 p_{1} p_{2} \simeq\left(p_{A}+p_{B}\right)^{2},
$$

where $s$ is supposed tending to infinity, and use the Sudakov decomposition of momenta

$$
p=\beta p_{1}+\alpha p_{2}+p_{\perp}, s \alpha \beta=p^{2}-p_{\perp}^{2}=p^{2}+\vec{p}^{2},
$$

where the vector sign denotes components of momenta transverse to the $p_{A}, p_{B}$ plane. They are supposed to be limited (not growing with $s$ ). 
According to the hypothesis of the gluon Reggeization the amplitude of the process $A+B \rightarrow A^{\prime}+B^{\prime}$ with a colour octet in the $t$-channel and negative signature (that means antisymmetry under the substitution $s \leftrightarrow u \simeq-s$ ) has the form:

$$
\mathcal{A}_{A B}^{A^{\prime} B^{\prime}}=\Gamma_{A^{\prime} A}^{c}\left[\left(\frac{-s}{-t}\right)^{j(t)}-\left(\frac{+s}{-t}\right)^{j(t)}\right] \Gamma_{B^{\prime} B}^{c},
$$

where

$$
t=q^{2} \simeq q_{\perp}^{2}=-\vec{q}^{2}, \quad q=p_{A}-p_{A^{\prime}}=p_{B^{\prime}}-p_{B} ; \quad j(t)=1+\omega(t) ;
$$

$j(t)$ is the gluon Regge trajectory, $\Gamma_{P^{\prime} P}^{c}$ are the vertices of the Reggeon interactions with scattered particles, $c$ is a colour index. The form (2.3) represents correctly the analytical structure of the scattering amplitude, which is quite simple in the elastic case. In the BFKL approach it is assumed that this form is valid in the NLA as well as in the LLA. Remind that in each order of perturbation theory amplitudes with negative signature do dominate, owing to the cancellation of the leading logarithmic terms in amplitudes with positive signatures, which become pure imaginary in the LLA due to this cancellation. Note that the amplitude of the process $A+B \rightarrow A^{\prime}+B^{\prime}$ can contain contributions of various colour states and signatures in the $t$-channel, so that, strictly speaking, we should indicate somehow in the L.H.S. (2.3) that only the contribution of a colour octet with negative signature is retained. But since in this paper we are interested only in such contributions, we have omitted this indication to simplify denotations. We do the same below considering the inelastic amplitudes, so that a colour octet and negative signature is always assumed, without explicit indication, in the channels with gluon quantum numbers.

In the leading order ( $\mathrm{LO}$ ) the vertices of the Reggeon interactions with quarks and gluons have very simple form in the helicity basis:

$$
\Gamma_{P^{\prime} P}^{c}=g T_{P^{\prime} P}^{c} \delta_{\lambda_{P^{\prime}} \lambda_{P}},
$$

where $g$ is the QCD coupling constant, $T_{P^{\prime} P}^{c}$ are the matrix elements of the colour group generators in corresponding representations and $\lambda$-s are helicities of the partons. But we'll need a basis-independent form of the vertices. For quarks with momenta $p$ and $p^{\prime}$ having predominant components along $p_{1}$ such form can be presented as

$$
\Gamma_{Q^{\prime} Q}^{c}=g \bar{u}\left(p^{\prime}\right) t^{c} \frac{\not p_{2}}{2 p p_{2}} u(p),
$$

where $t^{c}$ are the colour group generators in the fundamental representation; for antiquarks we have correspondingly

$$
\Gamma_{\bar{Q}^{\prime} \bar{Q}}^{c}=-g \bar{v}(p) t^{c} \frac{p_{2}}{2 p p_{2}} v\left(p^{\prime}\right) .
$$

For gluons with predominant components of momenta along $p_{1}$ we'll use physical polarization vectors $e(p) p=e\left(p^{\prime}\right) p^{\prime}=0$ in the light-cone gauge $e(p) p_{2}=e\left(p^{\prime}\right) p_{2}=0$, so that

$$
e(p)=e(p)_{\perp}-\frac{\left(e(p)_{\perp} p_{\perp}\right)}{p_{2} p} p_{2}, \quad e\left(p^{\prime}\right)=e\left(p^{\prime}\right)_{\perp}-\frac{\left(e\left(p^{\prime}\right)_{\perp} p_{\perp}^{\prime}\right)}{p_{2} p^{\prime}} p_{2}
$$

and

$$
\Gamma_{G^{\prime} G}^{c}=-g\left(e^{*}\left(p^{\prime}\right)_{\perp} e(p)_{\perp}\right) T_{G^{\prime} G}^{c},
$$




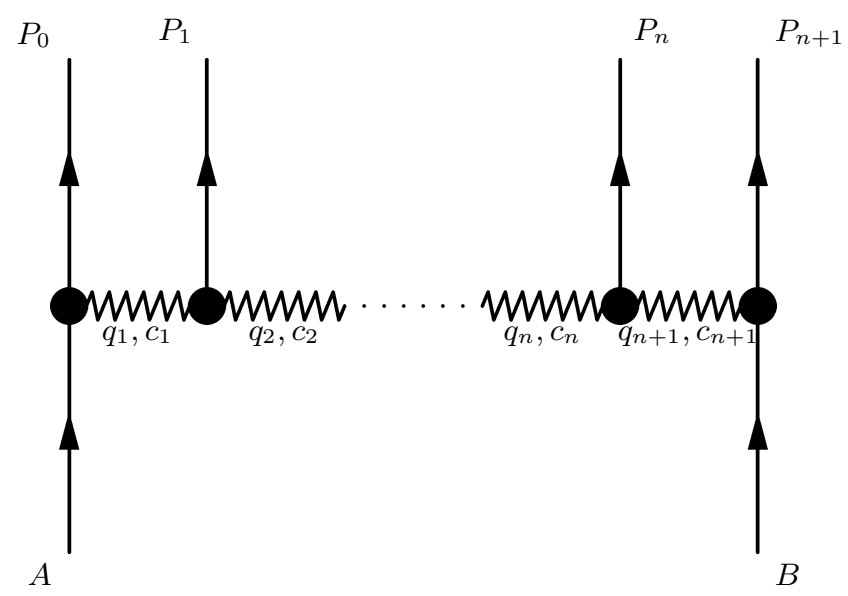

Figure 1: Schematic representation of the process $A+B \rightarrow P_{0}+P_{1}+\cdots+P_{n+1}$ in the MRK. The zig-zag lines represent Reggeized gluon exchange; the black circles denote the Regeon vertices; $q_{i}$ are the Reggeon momenta, flowing from the left to the right; $c_{i}$ are the colour indices.

with the colour generators in the adjoint representation. For momenta with predominant components along $p_{2}$ we have to replace in these formulas $p_{2} \rightarrow p_{1}$ (evidently, this replacement in (2.8) means change of the gauge). The gluon trajectory in the LO is given by

$$
\omega^{(1)}(t)=\frac{g^{2} N_{c} t}{2(2 \pi)^{D-1}} \int \frac{d^{D-2} q_{1}}{\vec{q}_{1}^{2}\left(\vec{q}-\vec{q}_{1}\right)^{2}}=-g^{2} \frac{N_{c} \Gamma(1-\epsilon)}{(4 \pi)^{D / 2}} \frac{\Gamma^{2}(\epsilon)}{\Gamma(2 \epsilon)}\left(\vec{q}^{2}\right)^{\epsilon} .
$$

Here and in the following $N_{c}$ is the number of colors, $D=4+2 \epsilon$ is the space-time dimension taken different from 4 to regularize infrared divergencies; $\Gamma(x)$ is the Eueler function.

The necessary assumption in the derivation of the BFKL equation is the Reggeized form of the production amplitudes in the multi-Regge kinematics (MRK), which means large invariant masses of any pair of final particles and fixed transferred momenta. Denoting momenta of final particles in the process $A+B \rightarrow P_{0}+P_{1}+\ldots+P_{n+1}$ as $k_{i}, i=0 \div n+1$ (see Fig. 1),

$$
k_{i}=\beta_{i} p_{1}+\alpha_{i} p_{2}+k_{i \perp}, \quad s \alpha_{i} \beta_{i}=k_{i}^{2}-k_{i \perp}^{2}=k_{i}^{2}+\vec{k}_{i}^{2},
$$

we can put in the MRK

$$
\alpha_{0} \ll \alpha_{1} \cdots \ll \alpha_{n} \ll \alpha_{n+1}, \quad \beta_{n+1} \ll \beta_{n} \cdots \ll \beta_{1} \ll \beta_{0} .
$$

Due to Eqs. (2.11) and (2.12) the squared invariant masses

$$
s_{i}=\left(k_{i-1}+k_{i}\right)^{2} \approx s \beta_{i-1} \alpha_{i}=\frac{\beta_{i-1}}{\beta_{i}}\left(k_{i}^{2}+\vec{k}_{i}^{2}\right)
$$

are large compared with the squared transverse momenta of produced particles, which are of order of the squared momentum transfers:

$$
s_{i} \gg \vec{k}_{i}^{2} \sim\left|t_{i}\right|=\left|q_{i}^{2}\right|,
$$

where

$$
q_{i}=p_{A}-\sum_{j=0}^{i-1} k_{j}=-\left(p_{B}-\sum_{j=i}^{n+1} k_{j}\right) \approx \beta_{i} p_{1}-\alpha_{i-1} p_{2}-\sum_{j=0}^{i-1} k_{j \perp},
$$




$$
t_{i}=q_{i}^{2} \approx q_{i \perp}^{2}=-\vec{q}_{i}^{2}
$$

and product of all $s_{i}$ is proportional to $s$ :

$$
\prod_{i=1}^{n+1} s_{i}=s \prod_{i=1}^{n}\left(k_{i}^{2}+\vec{k}_{i}^{2}\right)
$$

The production amplitudes have a complicated analytical structure (see, for instance, [30, [31]). Fortunately, only real parts of these amplitudes are used in the derivation of the BFKL equation in the NLA as well as in the LLA. We restrict ourselves also by consideration of the real parts, although it is not explicitly indicated below. They can be written as (see [12] and references therein)

$$
\begin{gathered}
\mathcal{A}_{A B}^{\tilde{A} \tilde{B}+n}=4\left(p_{A} p_{B}\right) \Gamma_{\tilde{A} A}^{c_{1}}\left[\prod_{i=1}^{n} \frac{1}{t_{i}} \gamma_{c_{i} c_{i+1}}^{P_{i}}\left(q_{i}, q_{i+1}\right)\left(\frac{s_{i}}{\sqrt{\vec{k}_{i-1}^{2} \vec{k}_{i}^{2}}}\right)^{\omega\left(t_{i}\right)}\right] \\
\times \frac{1}{t_{n+1}}\left(\frac{s_{n+1}}{\sqrt{\vec{k}_{n}^{2} \vec{k}_{n+1}^{2}}}\right)^{\omega\left(t_{n+1}\right)} \Gamma_{\tilde{B} B}^{c_{n+1}},
\end{gathered}
$$

where $\gamma_{c_{i} c_{i+1}}^{P_{i}}\left(q_{i}, q_{i+1}\right)$ are the so-called Reggeon-Reggeon-particle (RRP) vertices, i.e. the effective vertices for production of particles $P_{i}$ with momenta $k_{i}=q_{i}-q_{i+1}$ in the collision of the Reggeons with momenta $q_{i}$ and $-q_{i+1}$ and colour indices $c_{i}$ and $c_{i+1}$. In the MRK only gluons can be produced with the vertex

$$
\gamma_{c_{1} c_{2}}^{G}\left(q_{1}, q_{2}\right)=g T_{c_{1} c_{2}}^{a} e_{\mu}^{*}(k) C^{\mu}\left(q_{2}, q_{1}\right),
$$

where $a, k=q_{1}-q_{2}$ and $e(k)$ are respectively colour index, momentum and polarization vector of the gluon,

$$
\begin{gathered}
C^{\mu}\left(q_{2}, q_{1}\right)=-q_{1}^{\mu}-q_{2}^{\mu}+p_{1}^{\mu}\left(\frac{q_{1}^{2}}{k p_{1}}+2 \frac{k p_{2}}{p_{1} p_{2}}\right)-p_{2}^{\mu}\left(\frac{q_{2}^{2}}{k p_{2}}+2 \frac{k p_{1}}{p_{1} p_{2}}\right) \\
=-q_{1 \perp}^{\mu}-q_{2 \perp}^{\mu}-\frac{p_{1}^{\mu}}{2\left(k p_{1}\right)}\left(k_{\perp}^{2}-2 q_{1 \perp}^{2}\right)+\frac{p_{2}^{\mu}}{2\left(k p_{2}\right)}\left(k_{\perp}^{2}-2 q_{2 \perp}^{2}\right) .
\end{gathered}
$$

In the light cone gauge $e(k) p_{2}=0$ we have

$$
e_{\mu}^{*}(k) C^{\mu}\left(q_{2}, q_{1}\right)=-2 e_{\perp}^{*}(k)\left(q_{1 \perp}-k_{\perp} \frac{q_{1 \perp}^{2}}{k_{\perp}^{2}}\right) .
$$

In the NLA the multi-Regge form is assumed in the BFKL approach for the production amplitudes not only in the MRK, when all produced particles are strongly ordered in the rapidity space, but also in the QMRK, when a couple of two particles is produced with rapidities of the same order. The QMRK can be obtained replacing one of the particles $P_{i}$ in the MRK by this couple. Therefore the QMRK amplitudes have the same form (2.17) as 
in the MRK with one of the vertices $\gamma_{c_{i} c_{i+1}}^{P_{i}}$ or $\Gamma_{\tilde{P} P}^{c}$ substituted by a vertex for production of the couple.

If the particles $P_{1}$ and $P_{2}$ are produced in the fragmentation region of the particle $\mathrm{A}$, we have

$$
\mathcal{A}_{A B}^{\left\{P_{1} P_{2}\right\} B^{\prime}}=4\left(p_{A} p_{B}\right) \Gamma_{\left\{P_{1} P_{2}\right\} A}^{c} \frac{s^{\omega(t)}}{t} \Gamma_{B^{\prime} B}^{c}
$$

where now $q=p_{A}-k, \quad k=k_{1}+k_{2}, \quad k_{1}$ and $k_{2}$ are momenta of the particles $P_{1}$ and $P_{2}$ correspondingly; for their Sudakov parameters we have $\beta_{1} \sim \beta_{2} \sim 1, \beta_{1}+\beta_{2}=1, \quad \alpha_{1} \sim$ $\alpha_{2} \sim O(1 / s)$. The produced particles can be $g g$ or $q \bar{q}$ pair if the particle $A$ is the gluon and $q g$ when the particle $A$ is the quark.

If rapidities of components of the produced couple (it can be or $g g$ or $q \bar{q}$ pair) are far away from rapidities of colliding particles, then it is created by two Reggeized gluons, and its production is described by the vertices $\gamma_{c_{1} c_{2}}^{Q \bar{Q}}\left(q_{1}, q_{2}\right)$ or $\gamma_{c_{1} c_{2}}^{G_{1} G_{2}}\left(q_{1}, q_{2}\right)$, where $q_{1}, c_{1}$ and $-q_{2}, c_{2}$ are momenta and colour indices of the Reggeized gluons. The amplitude $\mathcal{A}_{A B}^{A^{\prime}\left\{P_{1} P_{2}\right\} B^{\prime}}$ describing production on the couple $P_{1}$ and $P_{2}$ with the Sudakov parameters $\alpha_{1} \sim \alpha_{2} \ll$ $1, \quad \beta_{1} \sim \beta_{2} \ll 1$, has the form

$$
\mathcal{A}_{A B}^{A^{\prime}\left\{P_{1} P_{2}\right\} B^{\prime}}=4\left(p_{A} p_{B}\right) \Gamma_{A^{\prime} A}^{c_{1}} \frac{s_{1}^{\omega\left(t_{1}\right)}}{t_{1}} \gamma_{c_{1} c_{2}}^{P_{1} P_{2}}\left(q_{1}, q_{2}\right) \frac{s_{2}^{\omega\left(t_{2}\right)}}{t_{2}} \Gamma_{B^{\prime} B}^{c_{2}}
$$

where

$$
\begin{gathered}
q_{1}=p_{A}-p_{A^{\prime}}, q_{2}=-p_{B}+p_{B^{\prime}}, t_{i}=q_{i}^{2} \simeq q_{i \perp}^{2}, \\
s_{1}=\left(p_{A^{\prime}}+k\right)^{2}, \quad s_{2}=\left(p_{B^{\prime}}+k\right)^{2}, k=k_{1}+k_{2}, \quad k^{2} \ll s_{1,2} \ll s .
\end{gathered}
$$

Note that because the QMRK in the unitarity relations leads to loss of the large logarithms, scales of energies in (2.21), (2.22) are unimportant in the NLA; moreover, the trajectory and the vertices are needed there only in the LO. The trajectory in this order is given by (2.10); the vertices are presented below. Remind that the vertices were extracted from corresponding amplitudes in the Born approximation, so that at the tree level Eqs.(2.21),(2.22) are verified. What has to be checked is their energy dependence, i.e. the Regge factors $s_{i}^{\omega\left(t_{i}\right)}$.

\section{Production in the fragmentation region}

\subsection{One-loop radiative corrections and bootstrap conditions}

To be definite, we consider below production in the fragmentation region of the particle $A$. In this section we use denotations $s_{1}=\left(p_{B^{\prime}}+p_{P_{1}}\right)^{2}$ and $s_{2}=\left(p_{B^{\prime}}+p_{P_{2}}\right)^{2}$. Note that here $s_{1} \sim s_{2} \sim s$, contrary to the case of production in the central region of rapidities. In the radiative corrections to the amplitude $\mathcal{A}_{A B}^{\left\{P_{1} P_{2}\right\} B^{\prime}}$ we have to retain only large logarithmic terms, not making difference between $\ln s, \ln s_{1}$ and $\ln s_{2}$. Therefore the corrections can be calculated using the $s$-channel unitarity in the same way as it was done for the elastic 


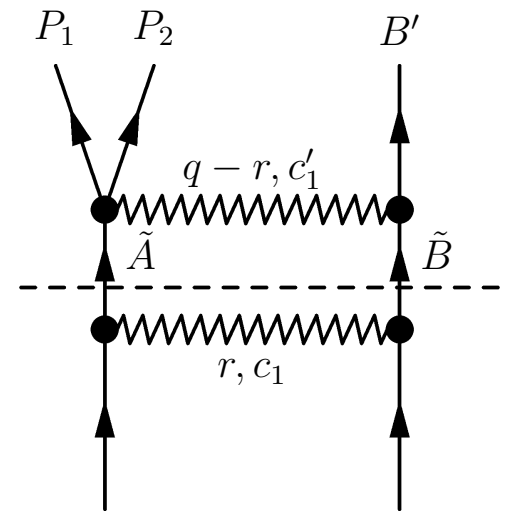

A
$B$

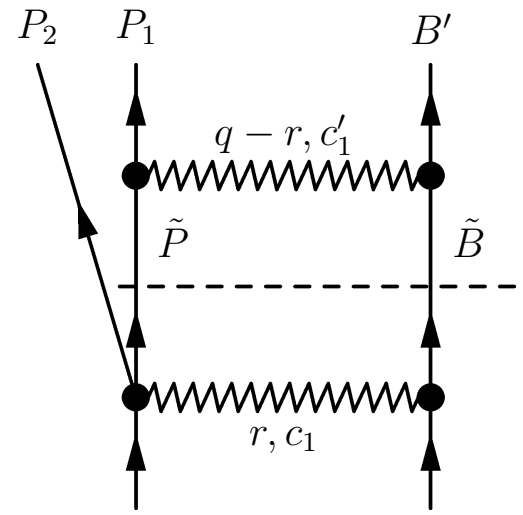

A

$B$

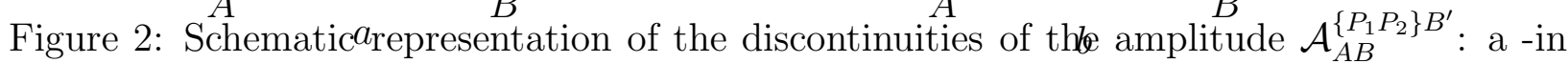
the $s$-channel; b - in the $s_{1}$-channel.

scattering amplitudes in the LLA [5]. The large logarithms are defined by the discontinuities of the amplitude $\mathcal{A}_{A B}^{\left\{P_{1} P_{2}\right\} B^{\prime}}$ in the channels $s, s_{1}$ and $s_{2}$, and we find them using the unitarity relations in these channels.

Let start with the $s$-channel discontinuity. In the one-loop approximation the intermediate states in the unitarity relation can be only two-particle states, so that we have (see Fig. 2 a)

$$
\Im_{s} \mathcal{A}_{A B}^{\left\{P_{1} P_{2}\right\} B^{\prime}}=\frac{1}{2} \sum_{\{\tilde{A} \tilde{B}\}} \int \mathcal{A}_{A B}^{\tilde{A} \tilde{B}} \mathcal{A}_{\tilde{A} \tilde{B}}^{\left\{P_{1} P_{2}\right\} B^{\prime}} d \Phi_{\tilde{A} \tilde{B}}
$$

where the sum $\sum_{\{\tilde{A} \tilde{B}\}}$ is over over all discrete quantum numbers of the particles $\tilde{A}$ and $\tilde{B}$, $d \Phi_{\tilde{A} \tilde{B}}$ is their phase space element. Here and in the following we use the Hermitian property of the Born amplitudes

$$
\left(\mathcal{A}_{i}^{f}\right)^{*}=\mathcal{A}_{f}^{i} .
$$

In the region which gives a leading (growing as $s$ ) contribution to the imaginary part

$$
d \Phi_{\tilde{A} \tilde{B}}=(2 \pi)^{D} \delta^{(D)}\left(p_{A}+p_{B}-p_{\tilde{A}}-p_{\tilde{B}}\right) \frac{d^{D-1} p_{\tilde{A}}}{2 \epsilon_{\tilde{A}}(2 \pi)^{D-1}} \frac{d^{D-1} p_{\tilde{B}}}{2 \epsilon_{\tilde{B}}(2 \pi)^{D-1}}=\frac{d^{D-2} r_{\perp}}{2 s(2 \pi)^{D-2}} .
$$

Here and below $r_{\perp}$ is the transverse part of the momentum transfer $p_{\tilde{B}}-p_{B}$. Note that for production in the fragmentation region the Sudakov parameters $\alpha$ and $\beta$ for the momentum transfer $p_{\tilde{B}}-p_{B}$ are $\sim 1 / s$, so that $p_{\tilde{B}}-p_{B} \simeq r_{\perp}$. For production in the central region it is not always correct.

The imaginary parts in the $s_{1,2}$-channels are calculated quite analogously. Take the $s_{1}$ channel. Denoting intermediate particles in the unitarity relation in this channel $\tilde{P}$ and $\tilde{B}$, we obtain (see Fig. 2 b)

$$
\Im_{\left(p_{B^{\prime}}+p_{P_{1}}\right)^{2}} \mathcal{A}_{A B}^{\left\{P_{1} P_{2}\right\} B^{\prime}}=\frac{1}{2} \sum_{\{\tilde{P} \tilde{B}\}} \int \mathcal{A}_{A B}^{\left\{\tilde{P} P_{2}\right\} \tilde{B}} \mathcal{A}_{\tilde{P} \tilde{B}}^{P_{1} B^{\prime}} d \Phi_{\tilde{P} \tilde{B}},
$$


with

$$
d \Phi_{\tilde{P} \tilde{B}}=(2 \pi)^{D} \delta^{(D)}\left(p_{P_{1}}+p_{B^{\prime}}-p_{\tilde{P}}-p_{\tilde{B}}\right) \frac{d^{D-1} p_{\tilde{P}}}{2 \epsilon_{\tilde{P}}(2 \pi)^{D-1}} \frac{d^{D-1} p_{\tilde{B}}}{2 \epsilon_{\tilde{B}}(2 \pi)^{D-1}}=\frac{d^{D-2} r_{\perp}}{2\left(p_{B^{\prime}}+p_{P_{1}}\right)^{2}(2 \pi)^{D-2}} .
$$

The $s_{2}$-channel imaginary part is obtained from (3.4), (3.5) by the substitution $P_{1} \leftrightarrow P_{2}$.

Since we don't make difference between $\ln s, \ln s_{1}$ and $\ln s_{2}$, we need only sum of the imaginary parts in the $s, s_{1}$ and $s_{2}$ channels. Using (2.3) and (2.21) in the Born approximation for the amplitudes in (3.1), (3.4) we obtain for the sum

$$
\Im \mathcal{A}_{A B}^{\left\{P_{1} P_{2}\right\} B^{\prime}}=\frac{s}{(2 \pi)^{D-2}} \int \frac{d^{D-2} r_{\perp}}{r_{\perp}^{2}(q-r)_{\perp}^{2}} \sum_{\{i\}} \Gamma_{\{i\} A}^{c_{1}} \Gamma_{\left\{P_{1} P_{2}\right\}\{i\}}^{c_{1}^{\prime}} \sum_{\{\tilde{B}\}} \Gamma_{\tilde{B} B}^{c_{1}} \Gamma_{B^{\prime} \tilde{B}}^{c_{1}^{\prime}},
$$

where the sum over $\{i\}$ is performed over all possible intermediate states and their quantum numbers. If $\{i\}$ contains two particles, one of them must be $P_{1}$ or $P_{2}$; in this case corresponding subscript in $\Gamma_{\left\{P_{1} P_{2}\right\}\{i\}}^{c^{\prime}}$ can be omitted.

Remind that we assume everywhere projection on a colour octet and negative signature in the $t$-channel. Performing this projection explicitly by the projection operator $\hat{\mathcal{P}}_{8_{a}}$,

$$
\left\langle c_{1} c_{1}^{\prime}\left|\hat{\mathcal{P}}_{8_{a}}\right| c_{2} c_{2}^{\prime}\right\rangle=\frac{f_{c_{1} c_{1}^{\prime} c} f_{c_{2} c_{2}^{\prime} c}}{N_{c}}
$$

where $f_{a b c}$ are the structure constants of the colour group, and using the bootstrap property of the $\mathrm{LO}$ vertices

$$
f_{c_{1} c_{1}^{\prime} c} \sum_{\{\tilde{B}\}} \Gamma_{\tilde{B} B}^{c_{1}} \Gamma_{B^{\prime} \tilde{B}}^{c_{1}^{\prime}}=-i g \frac{N_{c}}{2} \Gamma_{B^{\prime} B}^{c},
$$

which is easily derived from (2.5), we get

$$
\Im \mathcal{A}_{A B}^{\left\{P_{1} P_{2}\right\} B^{\prime}}=\frac{s}{t}\left(-\pi \frac{g t}{(2 \pi)^{D-1}} \int \frac{d^{D-2} r_{\perp}}{r_{\perp}^{2}(q-r)_{\perp}^{2}} i f_{c_{1} c_{1}^{\prime} c} \sum_{\{i\}} \Gamma_{\{i\} A}^{c_{1}}\left(r_{\perp}\right) \Gamma_{\left\{P_{1} P_{2}\right\}\{i\}}^{c_{1}^{\prime}}\left(q_{\perp}-r_{\perp}\right)\right) \Gamma_{B^{\prime} B}^{c} .
$$

Here we indicate explicitly dependence of the Reggeon vertices on momentum transfer. Remind that the sum over $\{i\}$ is performed over all possible intermediate states and their quantum numbers. All vertices here are taken in the leading order, so that if an intermediate state contains two particles, one of them must be the same as in the final state; another changes its transverse momentum and colour state, but its helicity is conserved. The real part of the one-loop contribution to the amplitude can be restored from the imaginary part by the substitution (cf.(2.3) )

$$
-\pi \rightarrow 2 \ln s
$$

Therefore, comparing (3.9) with the first order term in the expansion of (2.21) with account of (2.10), we see that the one-loop correction calculated above is compatible with the Reggeized form (2.21) only if

$$
\int \frac{d^{D-2} r_{\perp}}{r_{\perp}^{2}(q-r)_{\perp}^{2}} \frac{i f^{c c_{1} c_{1}^{\prime}}}{N_{c}} \sum_{\{i\}} \Gamma_{\{i\} A}^{c_{1}}\left(r_{\perp}\right) \Gamma_{\left\{P_{1} P_{2}\right\}\{i\}}^{c_{1}^{\prime}}\left(q_{\perp}-r_{\perp}\right)=\frac{g}{2} \Gamma_{\left\{P_{1} P_{2}\right\} A}^{c}\left(q_{\perp}\right) \int \frac{d^{D-2} r_{\perp}}{r_{\perp}^{2}(q-r)_{\perp}^{2}} .
$$


Eq. (3.11) gives the bootstrap conditions for the Reggeon vertices of two-particle production in the fragmentation region. In the next subsections we show that they are satisfied.

\subsection{Quark-antiquark production}

To produce a $q \bar{q}$ pair the particle $A$ must be a gluon. Let $p_{A}=p_{1}, a$ is the colour index of the initial gluon, $k_{1}$ and $k_{2}$ are the quark and antiquark momenta respectively,

$$
k_{1,2}=\beta_{1,2} p_{1}+\frac{m^{2}+\vec{k}_{1,2}^{2}}{s \beta_{1,2}} p_{2}+k_{1,2 \perp}, \quad k_{1 \perp}+k_{2 \perp}+q_{\perp}=0,
$$

$m$ is the quark mass. The intermediate states $\{i\}$ in (3.11) can be:

1) one-gluon state with momentum $p_{\tilde{A}}=p_{1}-r$;

2) $q \bar{q}$ state with quark and antiquark momenta $k_{1}^{\prime}=k_{1}+q-r$ and $k_{2}$ respectively;

3) $q \bar{q}$ state with quark and antiquark momenta respectively $k_{1}$ and $k_{2}^{\prime}=k_{2}+q-r$.

Apart from the "elastic" vertices (2.9),(2.6),(2.7) the bootstrap condition contains only the Reggeon vertex for $q \bar{q}$ production, which can be found in [15. In general case, when the pair is produced by the gluon $G$ with momentum $k=\beta p_{1}+\vec{k}^{2} /(\beta s) p_{2}+k_{\perp}$, the vertex can be presented as

$$
\begin{gathered}
\Gamma_{\{Q \bar{Q}\} G}^{c}=\left(t^{a} t^{c}\right)_{i_{1} i_{2}}\left(\mathcal{A}\left(\left(k_{1}-x_{1} k\right)_{\perp}\right)-\mathcal{A}\left(\left(x_{2} k_{1}-x_{1} k_{2}\right)_{\perp}\right)\right) \\
-\left(t^{c} t^{a}\right)_{i_{1} i_{2}}\left(\mathcal{A}\left(\left(-k_{2}+x_{2} k\right)_{\perp}\right)-\mathcal{A}\left(\left(x_{2} k_{1}-x_{1} k_{2}\right)_{\perp}\right)\right),
\end{gathered}
$$

where $x_{1,2}=\beta_{1,2} / \beta, \quad x_{1}+x_{2}=1, \quad i_{1}, i_{2}$ are quark and antiquark colour indices, $a$ is the colour index of the gluon $G$. The amplitudes $\mathcal{A}\left(p_{\perp}\right)$ in the light-cone gauge (2.8) are rather simple:

$$
\mathcal{A}\left(p_{\perp}\right)=\frac{g^{2}}{p_{\perp}^{2}-m^{2}} \bar{u}\left(k_{1}\right) \frac{p_{B}}{\beta s}\left(x_{1} \phi_{\perp} p_{\perp}-x_{2} p_{\perp} \phi_{\perp}-\phi_{\perp} m\right) v\left(k_{2}\right) .
$$

Here $e$ is the gluon polarization vector, $u\left(k_{1}\right)$ and $v\left(k_{2}\right)$ are the spin wave functions of the quark and antiquark respectively.

With the vertices $(2.9),(2.6),(2.7)$ and $(3.13)$ the contribution of either of the three intermediate states to the integrand in L.H.S. of (3.11) is readily calculated and we obtain correspondingly

1)

$$
\begin{gathered}
\frac{i g f^{c c_{1} c_{1}^{\prime}}}{N_{c}} T_{a^{\prime} a}^{c_{1}}\left[\left(t^{a^{\prime}} t^{c_{1}^{\prime}}\right)_{i_{1} i_{2}}\left(\mathcal{A}\left(\left(k_{1}+x_{1} r\right)_{\perp}\right)-\mathcal{A}\left(\left(x_{2} k_{1}-x_{1} k_{2}\right)_{\perp}\right)\right)\right. \\
\left.-\left(t^{c_{1}^{\prime}} t^{a^{\prime}}\right)_{i_{1} i_{2}}\left(\mathcal{A}\left(\left(-k_{2}-x_{2} r\right)_{\perp}\right)-\mathcal{A}\left(\left(x_{2} k_{1}-x_{1} k_{2}\right)_{\perp}\right)\right)\right]
\end{gathered}
$$

2)

$$
\begin{gathered}
\frac{i g f^{c c_{1} c_{1}^{\prime}}}{N_{c}}\left[\left(t^{c_{1}^{\prime}} t^{a} t^{c_{1}}\right)_{i_{1} i_{2}}\left(\mathcal{A}\left(\left(-k_{2}-r\right)_{\perp}\right)-\mathcal{A}\left(\left(-k_{2}-x_{2} r\right)_{\perp}\right)\right)\right. \\
\left.-\left(t^{c_{1}^{\prime}} t^{c_{1}} t^{a}\right)_{i_{1} i_{2}}\left(\mathcal{A}\left(\left(-k_{2}\right)_{\perp}\right)-\mathcal{A}\left(\left(-k_{2}-x_{2} r\right)_{\perp}\right)\right)\right]
\end{gathered}
$$


and

3)

$$
\begin{aligned}
& -\frac{i g f^{c c_{1} c_{1}^{\prime}}}{N_{c}}\left[\left(t^{a} t^{c_{1}} t^{c_{1}^{\prime}}\right)_{i_{1} i_{2}}\left(\mathcal{A}\left(\left(k_{1}\right)_{\perp}\right)-\mathcal{A}\left(\left(k_{1}+x_{1} r\right)_{\perp}\right)\right)\right. \\
& \left.-\left(t^{c_{1}} t^{a} t^{c_{1}^{\prime}}\right)_{i_{1} i_{2}}\left(\mathcal{A}\left(\left(k_{1}+r\right)_{\perp}\right)-\mathcal{A}\left(\left(k_{1}+x_{1} r\right)_{\perp}\right)\right)\right] .
\end{aligned}
$$

It's not difficult to see from these expressions that the terms with $\mathcal{A}\left(\left(k_{1}+x_{1} r\right)_{\perp}\right)$ are cancelled before integration, due to the commutation relations between $t^{i}$, as well as the terms with $\mathcal{A}\left(\left(-k_{2}-x_{2} r\right)_{\perp}\right)$. As for the terms with $\mathcal{A}\left(\left(k_{1}+r\right)_{\perp}\right)$ and $\mathcal{A}\left(\left(-k_{2}-r\right)_{\perp}\right)$, they cancel each other as a result of integration, due to invariance of the integration measure $d^{D-2} r_{\perp} /\left(r_{\perp}^{2}(q-r)_{\perp}^{2}\right)$ with respect to the substitution $\left(k_{1}+r\right)_{\perp} \leftrightarrow\left(-k_{2}-r\right)_{\perp}$, with account of $k_{1 \perp}+k_{2 \perp}+q_{\perp}=0$. A simple colour algebra shows that the remaining terms gather into $(g / 2) \Gamma_{\{Q \bar{Q}\} A}^{c}$, where $A$ is a gluon with momentum $p_{A}=p_{1}$ (see (3.13) ), that makes evident that the bootstrap condition (3.11) is satisfied.

\subsection{Two-gluon production}

The case of two-gluon production can be considered quite similarly. Again the particle $A$ must be a gluon. Using the same denotations as before, with the difference that $k_{1}$ and $k_{2}$ now are the momenta of the produced gluons (so that $m$ is replaced by 0 ), $i_{1}$ and $i_{2}$ are their colour indices. Denoting their polarization vectors in the light-cone gauge (2.8) $e_{1}$ and $e_{2}$, we can present the vertex $\Gamma_{\left\{G_{1} G_{2}\right\} G}^{c}$ of two-gluon production [15] in the same form as (3.13)

$$
\begin{gathered}
\Gamma_{\left\{G_{1} G_{2}\right\} G}^{c}=\left(T^{a} T^{c}\right)_{i_{1} i_{2}}\left(\mathcal{A}\left(\left(k_{1}-x_{1} k\right)_{\perp}\right)-\mathcal{A}\left(\left(x_{2} k_{1}-x_{1} k_{2}\right)_{\perp}\right)\right) \\
\quad-\left(T^{c} T^{a}\right)_{i_{1} i_{2}}\left(\mathcal{A}\left(\left(-k_{2}+x_{2} k\right)_{\perp}\right)-\mathcal{A}\left(\left(x_{2} k_{1}-x_{1} k_{2}\right)_{\perp}\right)\right)
\end{gathered}
$$

where the amplitudes $\mathcal{A}\left(p_{\perp}\right)$ now have the form:

$$
\mathcal{A}\left(p_{\perp}\right)=\frac{2 g^{2}}{p_{\perp}^{2}}\left[x_{1} x_{2}\left(e_{1 \perp}^{*} e_{2 \perp}^{*}\right)\left(e_{\perp} p_{\perp}\right)-x_{1}\left(e_{1 \perp}^{*} e_{\perp}\right)\left(e_{2 \perp}^{*} p_{\perp}\right)-x_{2}\left(e_{2 \perp}^{*} e_{\perp}\right)\left(e_{1 \perp}^{*} p_{\perp}\right)\right] .
$$

The intermediate states are now:

1) one-gluon state with gluon momentum $p_{\tilde{A}}=p_{1}-r$;

2) two-gluon state with gluon momenta $k_{1}^{\prime}=k_{1}+q-r$ and $k_{2}$;

3) two-gluon state with gluon momenta $k_{1}$ and $k_{2}^{\prime}=k_{2}+q-r$.

It is easy to see that the contributions of these states to the integrand in L.H.S. of (3.11) are given by the same formulas (3.15)-(3.17) as for the case of quark-antiquark production, with the only difference that the colour group generators are taken not in the fundamental, but in the adjoint representation. Since in the proof of fulfillment of the bootstrap conditions only the commutation relations of the generators were used, the proof can be applied to the case of two-gluon production as well as to $q \bar{q}$ production. 


\subsection{Quark-gluon production}

In the case of quark-gluon production (when the particle $A$ is a quark) the bootstrap condition can be considered in the same way. Let now $k$ is the momentum of incoming quark, $k_{1}$ and $k_{2}$ are the momenta of final quark and gluon correspondingly. Note that $k^{2}=k_{1}^{2}=m^{2}$, so that

$$
\begin{gathered}
k=\beta p_{1}+\frac{\vec{k}^{2}+m^{2}}{\beta s} p_{2}+k_{\perp}, \\
k_{1}=\beta_{1} p_{1}+\frac{\vec{k}_{1}^{2}+m^{2}}{\beta_{1} s} p_{2}+k_{1 \perp}, \quad k_{2}=\beta_{2} p_{1}+\frac{\vec{k}_{2}^{2}}{\beta_{2} s} p_{2}+k_{2 \perp} .
\end{gathered}
$$

Then from [16] one can obtain

$$
\begin{gathered}
\Gamma_{\{Q G\} Q}^{c}=\left(t^{a} t^{c}\right)_{i_{1} i_{2}}\left(\mathcal{A}\left(\left(x_{2} k_{1}-x_{1} k_{2}\right)_{\perp}\right)-\mathcal{A}\left(\left(k_{1}-x_{1} k\right)_{\perp}\right)\right) \\
-\left(t^{c} t^{a}\right)_{i_{1} i_{2}}\left(\mathcal{A}\left(\left(-k_{2}+x_{2} k\right)_{\perp}\right)-\mathcal{A}\left(\left(k_{1}-x_{1} k\right)_{\perp}\right)\right),
\end{gathered}
$$

where $i_{1}$ and $i_{2}$ are now the colour indices of the outgoing and incoming quarks, $a$ is the colour index of the produced gluon $G$, and the amplitudes $\mathcal{A}$ now have the form:

$$
\mathcal{A}\left(p_{\perp}\right)=-\frac{g^{2}}{p_{\perp}^{2}-x_{2}^{2} m^{2}} \bar{u}\left(k_{1}\right) \frac{p_{B}}{\beta s}\left(x_{1} \phi_{\perp}^{*} p_{\perp}+p_{\perp} \phi_{\perp}^{*}+\phi_{\perp}^{*} x_{2}^{2} m\right) u(p)
$$

Possible intermediate states are now:

1) one-quark state with quark momentum $p_{\tilde{A}}=p_{A}-r$; its contribution to the integrand in the L.H.S. of the bootstrap equation is

$$
\begin{aligned}
& \frac{i g f^{c c_{1} c_{1}^{\prime}}}{N_{c}}\left[\left(t^{a} t^{c_{1}^{\prime}} t^{c_{1}}\right)_{i_{1} i_{2}}\left(\mathcal{A}\left(\left(x_{2} k_{1}-x_{1} k_{2}\right)_{\perp}\right)-\mathcal{A}\left(\left(k_{1}+x_{1} r\right)_{\perp}\right)\right)\right. \\
& \left.\quad-\left(t^{c_{1}^{\prime}} t^{a} t^{c_{1}}\right)_{i_{1} i_{2}}\left(\mathcal{A}\left(\left(-k_{2}-x_{2} r\right)_{\perp}\right)-\mathcal{A}\left(\left(k_{1}+x_{1} r\right)_{\perp}\right)\right)\right] ;
\end{aligned}
$$

2) quark-gluon state with quark and gluon momenta $k_{1}^{\prime}=k_{1}+q-r$ and $k_{2}$ correspondingly; it gives

$$
\begin{gathered}
\frac{i g f^{c c_{1} c_{1}^{\prime}}}{N_{c}}\left[\left(t^{c^{\prime}} t^{a} t^{c_{1}}\right)_{i_{1} i_{2}}\left(\mathcal{A}\left(\left(-k_{2}-x_{2} r\right)_{\perp}\right)-\mathcal{A}\left(\left(-k_{2}-r\right)_{\perp}\right)\right)\right. \\
\left.-\left(t^{c_{1}^{\prime}} t^{c_{1}} t^{a}\right)_{i_{1} i_{2}}\left(\mathcal{A}\left(\left(-k_{2}\right)_{\perp}\right)-\mathcal{A}\left(\left(-k_{2}-r\right)_{\perp}\right)\right)\right]
\end{gathered}
$$

and

3) quark-gluon state with quark and gluon momenta $k_{1}$ and $k_{2}^{\prime}=k_{2}+q-r$; it contributes

$$
\begin{gathered}
\frac{i g f^{c c_{1} c_{1}^{\prime}}}{N_{c}} T_{a a^{\prime}}^{c_{1}^{\prime}}\left[\left(t^{a^{\prime}} t^{c_{1}}\right)_{i_{1} i_{2}}\left(\mathcal{A}\left(\left(k_{1}+x_{1} r\right)_{\perp}\right)-\mathcal{A}\left(\left(k_{1}\right)_{\perp}\right)\right)\right. \\
\left.-\left(t^{c_{1}} t^{a^{\prime}}\right)_{i_{1} i_{2}}\left(\mathcal{A}\left(\left(k_{1}+r\right)_{\perp}\right)-\mathcal{A}\left(\left(k_{1}\right)_{\perp}\right)\right)\right] .
\end{gathered}
$$


As well as in the case of $q \bar{q}$ production, it's not difficult to see that the terms with $\mathcal{A}\left(\left(k_{1}+x_{1} r\right)_{\perp}\right)$ and $\mathcal{A}\left(\left(-k_{2}-x_{2} r\right)_{\perp}\right)$ are cancelled before integration, due to colour algebra; the terms with $\mathcal{A}\left(\left(k_{1}+r\right)_{\perp}\right)$ and $\mathcal{A}\left(\left(-k_{2}-r\right)_{\perp}\right)$ cancel each other as a result of integration, and the remaining terms give $(g / 2) \Gamma_{\{G Q\} Q}^{c}$, where $Q$ is a quark with momentum $p_{A}=$ $p_{1}+\left(m^{2} / s\right) p_{2}($ see $(3.21))$.

It completes the proof that the bootstrap conditions (3.11) are satisfied.

We have considered here the case of $q g$ production. QCD invariance under the charge conjugation secures that the bootstrap condition is fulfilled also for $\bar{q} g$ production.

\section{Production in the central region}

\subsection{One-loop radiative corrections and bootstrap conditions}

Seing that only large logarithmic terms in the radiative corrections to the amplitude $\mathcal{A}_{A B}^{A^{\prime}\left\{P_{1} P_{2}\right\} B^{\prime}}$ must be retained, the corrections again can be calculated using the $s$-channel unitarity, as it was done for gluon production in the MRK in the LLA [5]. The logarithmic terms in the real part of the amplitude are obtained from the imaginary parts, connected with the discontinuities of the amplitude in channels with great (tending to infinity when $s \rightarrow \infty$ ) invariants, by the substitution (3.10), with corresponding invariant instead of $s$. Production of two particles with fixed invariant mass instead of one leads only to technical complications connected with existence of larger number of such invariants, analogously to the case of two particles in the fragmentation region compared with elastic scattering.

Let momenta of the produced particles $P_{1}$ and $P_{2}$ be $k_{1}$ and $k_{2}$ with $k_{1}+k_{2}=k=q_{1}-q_{2}$; $q_{1}=p_{A}-p_{A^{\prime}}$ and $q_{2}=p_{B^{\prime}}-p_{B}$ are transferred momenta; note that we can neglect by a component of $q_{1}\left(q_{2}\right)$ along $p_{2}\left(p_{1}\right)$, so that

$$
q_{1}=\beta p_{1}+q_{1 \perp}, \quad q_{2}=-\alpha p_{2}+q_{2 \perp}, \quad s \alpha \beta=\vec{k}^{2} .
$$

In the case of production of one particle with momentum $k$ in the MRK the large logarithms were defined by the discontinuities in the channels $s_{1}=\left(p_{A^{\prime}}+k\right)^{2}, s_{2}=\left(p_{B^{\prime}}+k\right)^{2}, s$ and $\left(p_{A^{\prime}}+p_{B^{\prime}}\right)^{2}$. Now we have more invariants which are great; but they can be divided into three groups of invariants of the same order $\left(\sim s_{1}, \sim s_{2}\right.$ and $\left.\sim s\right)$. Evidently, we have to calculate discontinuities in channels of all these invariants. Since we don't differ logarithms of invariants of one order, the real parts of the amplitude related to discontinuities in channels of invariants $\sim s_{a}\left(s_{a}\right.$ can be $s_{1}, s_{2}$ or $\left.s\right)$ are obtained from the imaginary parts by the substitution (3.10) with $s \rightarrow s_{a}$. Note that with our accuracy $\ln s=\ln s_{1}+\ln s_{2}$, therefore only two large logarithms in the real part can be considered as independent. We choose as independent $\ln s_{1}$ and $\ln s_{2}$. To calculate the contribution with $\ln s_{1}\left(\ln s_{2}\right)$ in the real part we have to find the sum of the imaginary parts in the channels with invariants of order $s_{1}$ $\left(s_{2}\right)$ and of order of $s$ and then to make the substitution (3.10) with $s_{1}\left(s_{2}\right)$ instead of $s$.

Therefore, to find the terms with $\ln s_{2}$ in the real part we need to calculate the imaginary parts in the channels $s_{2}=\left(p_{B^{\prime}}+k\right)^{2}, s_{21}=\left(p_{B^{\prime}}+k_{1}\right)^{2}, s_{22}=\left(p_{B^{\prime}}+k_{2}\right)^{2}, s=\left(p_{A}+p_{B}\right)^{2}$, 
$s^{\prime}=\left(p_{A^{\prime}}+p_{B^{\prime}}\right)^{2}, s_{1}^{\prime}=\left(p_{A^{\prime}}+k_{1}+p_{B^{\prime}}\right)^{2}$ and $s_{2}^{\prime}=\left(p_{A^{\prime}}+k_{2}+p_{B^{\prime}}\right)^{2}$, schematically shown in Figs. 3 a-g. Let us represent the sum of the imaginary parts as

$$
\Im \mathcal{A}_{A B}^{A^{\prime}\left\{P_{1} P_{2}\right\} B^{\prime}}=s \Gamma_{A^{\prime} A}^{c_{1}} \frac{1}{t_{1}}\left(-\pi \frac{g t_{2}}{(2 \pi)^{D-1}} \int \frac{d^{D-2} r_{\perp}}{r_{\perp}^{2}\left(q_{2}-r\right)_{\perp}^{2}} \mathcal{F}_{c_{1} c_{2}}^{P_{1} P_{2}}\left(q_{1}, q_{2}, r_{\perp}\right)\right) \frac{1}{t_{2}} \Gamma_{B^{\prime} B}^{c_{2}} .
$$

Below a possibility of such representation (which could be clear for an advanced reader) is shown and the contributions to $\mathcal{F}_{c_{1} c_{2}}^{P_{1} P_{2}}\left(q_{1}, q_{2}, r_{\perp}\right)$ from the imaginary parts in each of the channels are found. Let start with the $s_{2}$-channel (see Fig. 3 a):

$$
\Im_{3 a} \mathcal{A}_{A B}^{A^{\prime}\left\{P_{1} P_{2}\right\} B^{\prime}}=\frac{1}{2} \sum_{\{\tilde{P} \tilde{B}\}} \int \mathcal{A}_{A B}^{A^{\prime} \tilde{P} \tilde{B}} \mathcal{A}_{\tilde{P} \tilde{B}}^{\left\{P_{1} P_{2}\right\} B^{\prime}} d \Phi_{\tilde{P} \tilde{B}}
$$

where $d \Phi_{\tilde{P} \tilde{B}}$ is given by (3.5) with the replacement $p_{P_{1}} \rightarrow k$. As always, $r_{\perp}=\left(p_{\tilde{B}}-p_{B}\right)_{\perp}$. The particle $\tilde{P}$ has to be produced in the MRK, so that it must be a gluon. Denoting its momentum $k^{\prime}$ we have

$$
k^{\prime}=\beta p_{1}-\frac{\left(q_{1}-r\right)_{\perp}^{2}}{\beta s} p_{2}+\left(q_{1}-r\right)_{\perp}
$$

The possibility of the representation (4.2) for the imaginary part (4.3) becomes evident if one takes the representations (2.17) and (2.21) in the Born approximation for the amplitudes in (4.3), extracts the antisymmetric colour octet in the $t_{2}$-channel $\left(t_{2}=\left(p_{B}-p_{B^{\prime}}\right)^{2}\right)$ by the projection operator (3.7) and uses the bootstrap property of the LO vertices (3.8). For the contribution $\mathcal{F}_{c_{1} c_{2}}^{a}$ to $\mathcal{F}_{c_{1} c_{2}}^{P_{1} P_{2}}\left(q_{1}, q_{2}, r_{\perp}\right)$ one obtains

$$
\mathcal{F}_{c_{1} c_{2}}^{a}=i f_{i j c_{2}} \sum_{\{G\}} \gamma_{c_{1} i}^{G}\left(q_{1}, q_{1}-k^{\prime}\right) \Gamma_{\left\{P_{1} P_{2}\right\} G}^{j}
$$

Imaginary parts in the channels $\left(p_{B^{\prime}}+k_{1}\right)^{2}$ and $\left(p_{B^{\prime}}+k_{2}\right)^{2}$ (see Fig. 3 b,c) are found quite analogously. For the first of them we have

$$
\Im_{3 b} \mathcal{A}_{A B}^{A^{\prime}\left\{P_{1} P_{2}\right\} B^{\prime}}=\frac{1}{2} \sum_{\{\tilde{P} \tilde{B}\}} \int \mathcal{A}_{A B}^{A^{\prime}\left\{\tilde{P} P_{2}\right\} \tilde{B}} \mathcal{A}_{\tilde{P} \tilde{B}}^{P_{1} B^{\prime}} d \Phi_{\tilde{P} \tilde{B}}
$$

where $d \Phi_{\tilde{P} \tilde{B}}$ is given now just by (3.5). Evidently, the particle $\tilde{P}$ now is of the same kind as $P_{1}$. Denoting its momentum $k_{1}^{\prime}$ we have

$$
k_{1}^{\prime}=\beta_{1} p_{1}+\frac{m_{1}^{2}-\left(q_{1}-k_{2}-r\right)_{\perp}^{2}}{\beta_{1} s} p_{2}+\left(q_{1}-k_{2}-r\right)_{\perp},
$$

where $m_{1}$ is its mass. The amplitudes $\mathcal{A}_{A B}^{A^{\prime}\left\{\tilde{P} P_{2}\right\} \tilde{B}}$ and $\mathcal{A}_{\tilde{P} \tilde{B}}^{P_{1} B^{\prime}}$ are given by (2.22) and (2.3) respectively, taken in the Born approximation. After extraction of the antisymmetric colour octet in the $t_{2}$-channel and use of (3.8) we come to the representation (4.2) with the contribution $\mathcal{F}_{c_{1} c_{2}}^{b}$ to $\mathcal{F}_{c_{1} c_{2}}^{P_{1} P_{2}}\left(q_{1}, q_{2}, r_{\perp}\right)$ equal

$$
\mathcal{F}_{c_{1} c_{2}}^{b}=i f_{i j c_{2}} \sum_{\{\tilde{P}\}} \gamma_{c_{1} i}^{\tilde{P} P_{2}}\left(q_{1}, q_{1}-k_{1}^{\prime}-k_{2}\right) \Gamma_{P_{1} \tilde{P}}^{j} .
$$



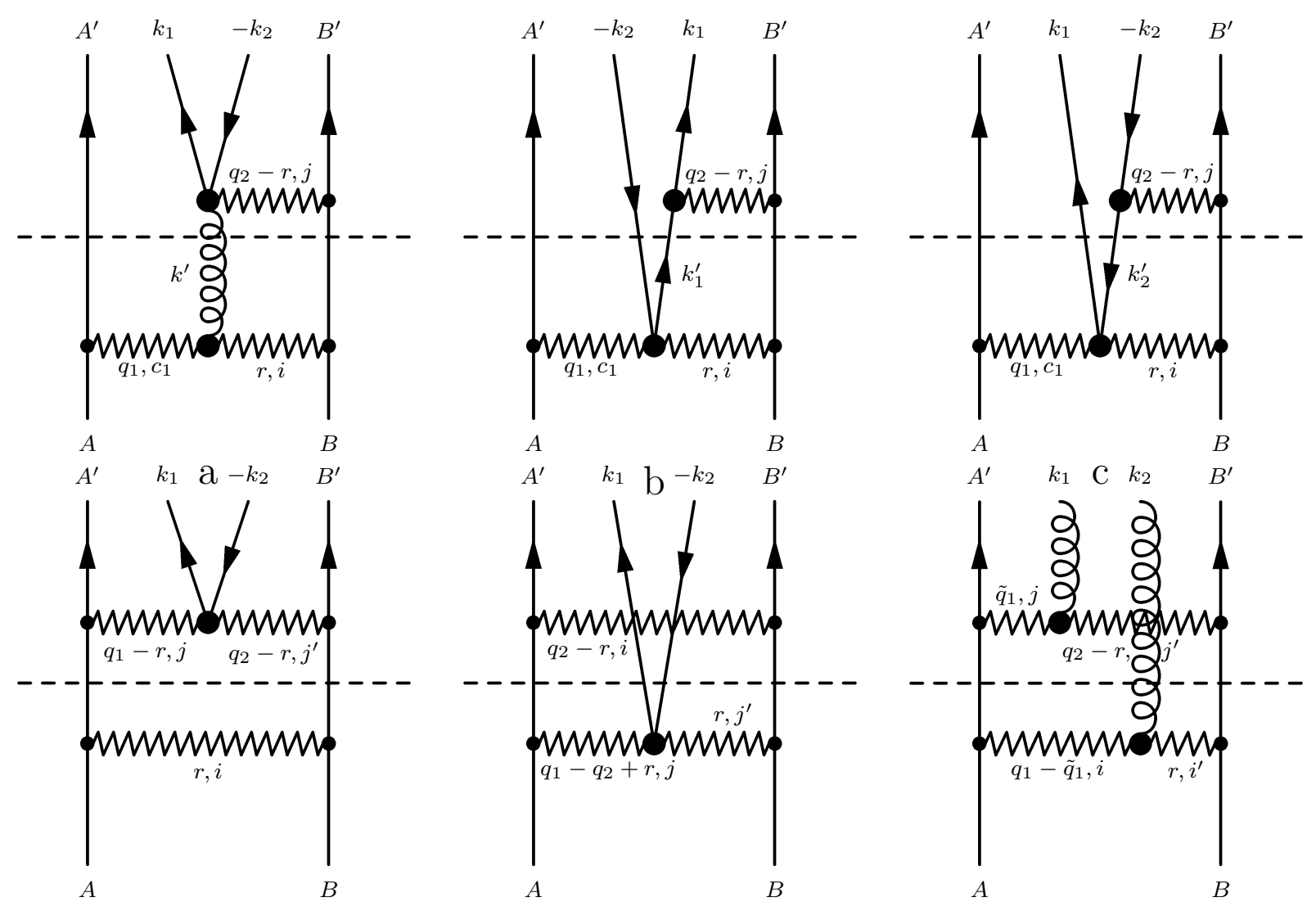

d

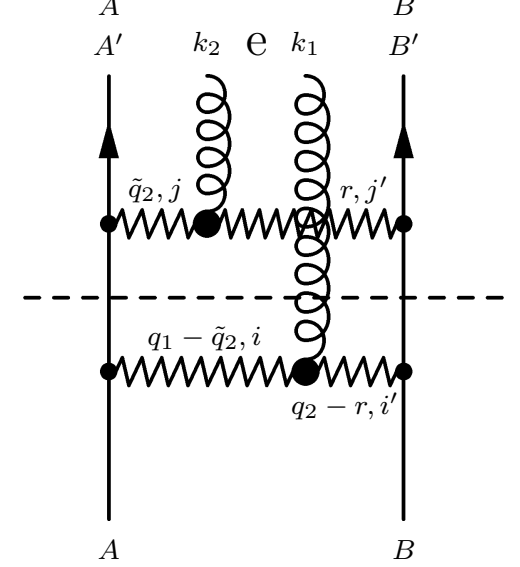

Figure 3: Schematic representation of the discontinuities of the amplitude $\mathcal{A}_{A B}^{A^{\prime}\left\{P_{1} P_{2}\right\} B^{\prime}}$ : a -in the $s_{2}$-channel; b -in the $s_{21}$-channel; c -in the $s_{22}$-channel; d -in the $s$-channel; e -in the $s^{\prime}$-channel; f -in the $s_{1}^{\prime}$-channel ; g -in the $s_{2}^{\prime}$-channel. 
Evidently,

$$
\mathcal{F}_{c_{1} c_{2}}^{c}=\mathcal{F}_{c_{1} c_{2}}^{b}\left(P_{1} \leftrightarrow P_{2}\right) .
$$

The imaginary parts shown in Figs. 3 d-g are calculated in a similar way. For Fig. 3 d one has

$$
\Im_{3 d} \mathcal{A}_{A B}^{A^{\prime}\left\{P_{1} P_{2}\right\} B^{\prime}}=\frac{1}{2} \sum_{\{\tilde{A} \tilde{B}\}} \int \mathcal{A}_{A B}^{\tilde{A} \tilde{B}} \mathcal{A}_{\tilde{A} \tilde{B}}^{A^{\prime}\left\{P_{1} P_{2}\right\} B^{\prime}} d \Phi_{\tilde{A} \tilde{B}}
$$

where $d \Phi_{\tilde{A} \tilde{B}}$ is given by (3.3); $; r_{\perp}=\left(p_{\tilde{B}}-p_{B}\right)_{\perp} \simeq p_{\tilde{B}}-p_{B}$. The amplitudes $\mathcal{A}_{A B}^{\tilde{A} \tilde{B}}$ and $\mathcal{A}_{\tilde{P} \tilde{B}}^{A^{\prime}\left\{\tilde{P} P_{2}\right\} B^{\prime}}$ are given by the Born terms of (2.22) and (2.3) respectively. The difference of further calculation from preceding ones is that it is necessary to apply the projection operator (3.7) and to use the bootstrap property (3.8) both in the $t_{1}$ - and $t_{2}$-channels. After this it becomes clear that again the imaginary parts have the form (4.2) with the contribution to $\mathcal{F}_{c_{1} c_{2}}^{P_{1} P_{2}}\left(q_{1}, q_{2}, r_{\perp}\right)$ equal

$$
\mathcal{F}_{c_{1} c_{2}}^{d}=\frac{g}{2} f_{i j c_{1}} f_{i j^{\prime} c_{2}} \frac{q_{1 \perp}^{2}}{\left(q_{1}-r\right)_{\perp}^{2}} \gamma_{j j^{\prime}}^{P_{1} P_{2}}\left(q_{1}-r_{\perp}, q_{2}-r_{\perp}\right) .
$$

The imaginary part answering Fig. 3 e is

$$
\Im_{3 e} \mathcal{A}_{A B}^{A^{\prime}\left\{P_{1} P_{2}\right\} B^{\prime}}=\frac{1}{2} \sum_{\{\tilde{A} \tilde{B}\}} \int \mathcal{A}_{A B}^{\tilde{A}\left\{P_{1} P_{2}\right\} \tilde{B}} \mathcal{A}_{\tilde{A} \tilde{B}}^{A^{\prime} B^{\prime}} d \Phi_{\tilde{A} \tilde{B}}
$$

where $d \Phi_{\tilde{A} \tilde{B}}$ is given now by (3.3) with the replacement $\left(p_{A}+p_{B} \rightarrow p_{A^{\prime}}+p_{B^{\prime}}\right)$. It is easy to see that the contribution of this imaginary part to $\mathcal{F}_{c_{1} c_{2}}^{P_{1} P_{2}}\left(q_{1}, q_{2}, r_{\perp}\right)$ is obtained from $\mathcal{F}_{c_{1} c_{2}}^{d}$ by the substitution $r \leftrightarrow q_{2}-r$. Since the integration measure in (4.2) is invariant under this substitution, we can put

$$
\mathcal{F}_{c_{1} c_{2}}^{e}=\mathcal{F}_{c_{1} c_{2}}^{d} .
$$

At last, Figs. 3 f,g appear only in the case when the particles $P_{1}$ and $P_{2}$ are gluons. The imaginary part answering Fig. $3 \mathrm{f}$ is

$$
\Im_{3 f} \mathcal{A}_{A B}^{A^{\prime}\left\{P_{1} P_{2}\right\} B^{\prime}}=\frac{1}{2} \sum_{\{\tilde{A} \tilde{B}\}} \int \mathcal{A}_{A B}^{\tilde{A} P_{2} \tilde{B}} \mathcal{A}_{\tilde{A} \tilde{B}}^{A^{\prime} P_{1} B^{\prime}} d \Phi_{\tilde{A} \tilde{B}}
$$

The amplitudes entering in (4.14) are given by (2.17) with $n=1$ in the Born approximation. Again applying the projection operator (3.7) and using the bootstrap property (3.8) in the $t_{1}$ - and $t_{2}$-channels we obtain

$$
\mathcal{F}_{c_{1} c_{2}}^{f}=\frac{g}{2} f_{i^{\prime} j^{\prime} c_{2}} f_{i j c_{1}} \frac{q_{1 \perp}^{2}}{\tilde{q}_{1 \perp}^{2}\left(q_{1}-\tilde{q}_{1}\right)_{\perp}^{2}} \gamma_{i i^{\prime}}^{P_{2}}\left(q_{1}-\tilde{q}_{1}, q_{1}-\tilde{q}_{1}-k_{2}\right) \gamma_{j j^{\prime}}^{P_{1}}\left(\tilde{q}_{1}, \tilde{q}_{1}-k_{1}\right),
$$

where $\tilde{q}_{1}=\beta_{1} p_{1}+\left(k_{1}+q_{2}-r\right)_{\perp}$. Evidently,

$$
\mathcal{F}_{c_{1} c_{2}}^{g}=\mathcal{F}_{c_{1} c_{2}}^{f}\left(P_{1} \leftrightarrow P_{2}\right) .
$$

Note that $\mathcal{F}_{c_{1} c_{2}}^{f}$ is invariant under simultaneous substitution $P_{1} \leftrightarrow P_{2}$ (that means, in particular, $\left.k_{1} \leftrightarrow k_{2}\right)$ and $r_{\perp} \leftrightarrow\left(q_{2}-r\right)_{\perp}$. The last substitution can be considered as the 
redefinition of $r_{\perp}$. Since the integration measure in (4.2) is invariant under this redefinition, we can make

$$
\mathcal{F}_{c_{1} c_{2}}^{g}=\mathcal{F}_{c_{1} c_{2}}^{f}
$$

Therefore, we have

$$
\mathcal{F}_{c_{1} c_{2}}^{P_{1} P_{2}}\left(q_{1}, q_{2}, r\right)=\mathcal{F}_{c_{1} c_{2}}^{a}+\mathcal{F}_{c_{1} c_{2}}^{b}+\mathcal{F}_{c_{1} c_{2}}^{c}+2 \mathcal{F}_{c_{1} c_{2}}^{d}+2 \mathcal{F}_{c_{1} c_{2}}^{f}
$$

where the terms in the R.H.S. are given respectively by Eqs. (4.5), (4.8), (4.9), (4.11) and (4.15).

As it was discussed earlier, the terms with $\ln s_{2}$ in the real part of the amplitude $\mathcal{A}_{A B}^{A^{\prime}\left\{P_{1} P_{2}\right\} B^{\prime}}$ are obtained from (4.2) by the substitution (3.10) with $s_{2}$ instead of $s$. Comparing the obtained result with (2.22) with account of (2.10), we see that the one-loop correction calculated above is compatible with the Reggeized form (2.22) only if

$$
\int \frac{d^{D-2} r_{\perp}}{r_{\perp}^{2}\left(q_{2}-r\right)_{\perp}^{2}} \mathcal{F}_{c_{1} c_{2}}^{P_{1} P_{2}}\left(q_{1}, q_{2}, r_{\perp}\right)=\frac{g N_{c}}{2} \gamma_{c_{1} c_{2}}^{\left\{P_{1} P_{2}\right\}}\left(q_{1}, q_{2}\right) \int \frac{d^{D-2} r_{\perp}}{r_{\perp}^{2}\left(q_{2}-r\right)_{\perp}^{2}}
$$

Eq.(4.19) gives the bootstrap conditions for the vertices of pair production in ReggeonReggeon collisions. They are verified in the next subsections.

\subsection{Quark-antiquark production}

For simplicity, we discuss below the case of the massless quarks, although the massive case can be considered quite analogously.

\section{Denotations}

Remind that $k_{1}$ and $k_{2}$ are the quark and antiquark momenta respectively;

$$
\begin{gathered}
k_{i}=\beta_{i} p_{1}+\alpha_{i} p_{2}+k_{i \perp}, \quad i=1,2, \quad s \alpha_{i} \beta_{i}=-k_{i \perp}^{2}=\vec{k}_{i}^{2} ; \\
\beta_{i}=x_{i} \beta, \quad \beta=\beta_{1}+\beta_{2} ; k=k_{1}+k_{2}=q_{1}-q_{2}
\end{gathered}
$$

and we can put

$$
q_{1}=\beta p_{1}+q_{1 \perp}, q_{2}=-\alpha p_{2}+q_{2 \perp}, \beta=\beta_{1}+\beta_{2}, \alpha=\alpha_{1}+\alpha_{2} .
$$

We use also

$$
\begin{gathered}
k^{\prime}=\beta p_{1}-\frac{\left(q_{1}-r\right)_{\perp}^{2}}{\beta s} p_{2}+\left(q_{1}-r\right)_{\perp}, \quad k_{1}^{\prime}=\beta_{1} p_{1}-\frac{\left(q_{1}-k_{2}-r\right)_{\perp}^{2}}{\beta_{1} s} p_{2}+\left(q_{1}-k_{2}-r\right)_{\perp}, \\
k_{2}^{\prime}=\beta_{2} p_{1}-\frac{\left(q_{1}-k_{1}-r\right)_{\perp}^{2}}{\beta_{2} s} p_{2}+\left(q_{1}-k_{1}-r\right)_{\perp} .
\end{gathered}
$$


The function $\mathcal{F}_{c_{1} c_{2}}^{P_{2}}\left(q_{1}, q_{2}, r_{\perp}\right)$ in (4.19) is expressed in terms of the Reggeon vertices defined in (2.6) , (2.7), (2.18), (3.13) and the effective vertex of quark-antiquark production in ReggeonReggeon collisions. The last vertex was found in 32 and has the form

$$
\gamma_{c_{1} c_{2}}^{Q \bar{Q}}\left(q_{1}, q_{2}\right)=\frac{1}{2} g^{2} \bar{u}\left(k_{1}\right)\left[t^{c_{1}} t^{c_{2}} a\left(q_{1} ; k_{1}, k_{2}\right)-t^{c_{2}} t^{c_{1}} \overline{a\left(q_{1} ; k_{2}, k_{1}\right)}\right] v\left(k_{2}\right),
$$

where $a\left(q_{1} ; k_{1}, k_{2}\right)$ and $\overline{a\left(q_{1} ; k_{2}, k_{1}\right)}$ can be written [33] in the following way:

$$
a\left(q_{1} ; k_{1}, k_{2}\right)=\frac{4 p_{1} \emptyset_{1} p_{2}}{s \tilde{t}_{1}}-\frac{1}{k^{2}} \text { If }, \overline{a\left(q_{1} ; k_{2}, k_{1}\right)}=\frac{4 p_{2} \emptyset_{2} p_{1}}{s \tilde{t}_{2}}-\frac{1}{k^{2}} \text { If },
$$

with

$$
\begin{gathered}
\tilde{t}_{1}=\left(q_{1}-k_{1}\right)^{2}, \quad \tilde{t}_{2}=\left(q_{1}-k_{2}\right)^{2}, \quad Q_{1}=q_{1 \perp}-k_{1 \perp}, \quad Q_{2}=q_{1 \perp}-k_{2 \perp}, \\
\Gamma=2\left[\left(q_{1}+q_{2}\right)_{\perp}-\beta p_{A}\left(1-2 \frac{\vec{q}_{1}^{2}}{s \alpha \beta}\right)+\alpha p_{B}\left(1-2 \frac{\vec{q}_{2}^{2}}{s \alpha \beta}\right)\right] .
\end{gathered}
$$

Further for denominators in the Reggeon vertices we use denotations $D(p, q)$ and $d(p, q)$ :

$$
D(p, q)=x_{1} p_{\perp}^{2}+x_{2} q_{\perp}^{2}, \quad d(p, q)=\left(x_{1} p_{\perp}-x_{2} q_{\perp}\right)^{2} ; \quad D(p, q)=d(p, q)+x_{1} x_{2}\left(p_{\perp}+q_{\perp}\right)^{2} .
$$

Seeing that for arbitrary $p_{\perp}$

$$
\bar{u}\left(k_{1}\right) \not p_{\perp} v\left(k_{2}\right)=\bar{u}\left(k_{1}\right) \frac{\not p_{2}}{s \beta}\left(\frac{\not k_{1} \perp p_{\perp}}{x_{1}}+\frac{\not p_{\perp} \not k_{2 \perp}}{x_{2}}\right) v\left(k_{2}\right)
$$

we can present $a\left(q_{1} ; k_{1}, k_{2}\right)$ and $\overline{a\left(q_{1} ; k_{2}, k_{1}\right)}$ as

$$
a\left(q_{1} ; k_{1}, k_{2}\right)=\frac{4}{s \beta} p_{2} b\left(q_{1} ; k_{1}, k_{2}\right), \overline{a\left(q_{1} ; k_{2}, k_{1}\right)}=\frac{4}{s \beta} p_{2} \overline{b\left(q_{1} ; k_{2}, k_{1}\right)},
$$

where

$$
\begin{gathered}
b\left(q_{1} ; k_{1}, k_{2}\right)=\frac{\not k_{1 \perp}\left(\not k_{1 \perp}-\not q_{1 \perp}\right)}{D\left(k_{1}-q_{1}, k_{1}\right)}-\frac{x_{1} x_{2}}{d\left(k_{2}, k_{1}\right)}\left(\frac{q_{1 \perp}^{2} \not k_{1 \perp} \not k_{2 \perp}}{D\left(k_{2}, k_{1}\right)}\right. \\
-\frac{\not k_{1 \perp} \not q_{1 \perp}}{x_{1}}-\frac{\not q_{1 \perp} \not k_{2 \perp}}{x_{2}}-q_{1 \perp}^{2}+2\left(q_{1 \perp}\left(k_{1}+k_{2}\right) \perp\right)-1, \\
\overline{b\left(q_{1} ; k_{2}, k_{1}\right)}=\frac{\left(\not k_{2 \perp}-\not q_{1 \perp}\right) \not k_{2} \perp}{D\left(k_{2}, k_{2}-q_{1}\right)}-\frac{x_{1} x_{2}}{d\left(k_{2}, k_{1}\right)}\left(\frac{q_{1 \perp}^{2} \not k_{1 \perp} \not k_{2} \perp}{D\left(k_{2}, k_{1}\right)}\right. \\
-\frac{\not k_{1 \perp} \not q_{1 \perp}}{x_{1}}-\frac{\not q_{1 \perp} \not k_{2 \perp}}{x_{2}}-q_{1 \perp}^{2}+2\left(q_{1 \perp}\left(k_{1}+k_{2}\right) \perp\right)-1 .
\end{gathered}
$$

This form of $a\left(q_{1} ; k_{1}, k_{2}\right)$ and $\overline{a\left(q_{1} ; k_{2}, k_{1}\right)}$ permits to perform quite readily the summation over spin projections $\lambda$ of intermediate quarks and antiquarks in the contributions $\mathcal{F}_{c_{1} c_{2}}^{b}$ and $\mathcal{F}_{c_{1} c_{2}}^{c}$ to $\mathcal{F}_{c_{1} c_{2}}^{P_{1} P_{2}}\left(q_{1}, q_{2}, r_{\perp}\right)$; for example:

$$
\sum_{\lambda} \bar{u}\left(k_{1}\right) \frac{p_{2}}{\beta_{1} s} u^{\lambda}\left(k_{1}^{\prime}\right) \bar{u}^{\lambda}\left(k_{1}^{\prime}\right) a\left(q_{1} ; k_{1}^{\prime}, k_{2}\right) v\left(k_{2}\right)=\bar{u}\left(k_{1}\right) a\left(q_{1}, k_{1}^{\prime}, k_{2}\right) v\left(k_{2}\right) .
$$




\section{Independent colour structures}

It is easy to calculate the number of independent colour structures for production of a $q \bar{q}$ pair by two Reggeized gluons. Indeed, the pair can be either in a colour singlet, or in a colour octet state. Due to the colour symmetry each of these state can be produced only by the same state of two Reggeized gluons, which are colour octets. Since there is one singlet and two octets (symmetric and antisymmetric) in decomposition of product of two octets into irreducible representations, the number of independent colour structures is three. Their choice is not unique. We accept the following one:

$$
\mathcal{R}_{1}^{c_{1} c_{2}}=\frac{1}{N_{c}} f^{c_{1} i a} f^{c_{2} i b}\left(t^{a} t^{b}+t^{b} t^{a}\right), \quad \mathcal{R}_{2}^{c_{1} c_{2}}=i f^{c_{1} c_{2} i} t^{i}, \quad \mathcal{R}_{3}^{c_{1} c_{2}}=t^{c_{1}} t^{c_{2}}+t^{c_{2}} t^{c_{1}}
$$

From the equality

$$
t^{a} t^{b}=\frac{1}{2 N_{c}} \delta^{a b}+\frac{1}{2} d^{a b c} t^{c}+\frac{1}{2} i f^{a b c} t^{c}
$$

it is seen that the first and the third structures contain a singlet and a symmetric octet, whereas the second structure contains only an antisymmetric octet.

\section{Representation of $\mathcal{F}_{c_{1} c_{2}}^{P_{1} P_{2}}\left(q_{1}, q_{2}, r\right)$}

Using these colour structure we can represent each of the contribution $\mathcal{F}_{c_{1} c_{2}}^{i}$ entering in $\mathcal{F}_{c_{1} c_{2}}^{P_{1} P_{2}}\left(q_{1}, q_{2}, r\right)$ (4.18) in the form

$$
\mathcal{F}_{c_{1} c_{2}}^{i}=\frac{g^{3} N_{c}}{s \beta} \bar{u}\left(k_{1}\right) p_{2} \sum_{n=1}^{n=3} \mathcal{R}_{n}^{c_{1} c_{2}} \mathcal{L}_{n}^{i} v\left(k_{2}\right)
$$

It is not difficult to find all $\mathcal{L}_{n}^{i}$ from the equations presented above.

From (4.5), using the Reggeon-Reggeon-gluon (RRG) vertex (2.18) in the gauge (2.20) and the vertex for $q \bar{q}$ production in the fragmentation region (3.13) we obtain:

$$
\begin{gathered}
\mathcal{L}_{1}^{a}=\frac{x_{1} \not \not_{1 \perp}\left(\not k_{1 \perp}-x_{1} \not k_{\perp}^{\prime}\right)-x_{2}\left(\not k_{1 \perp}-x_{1} \not k_{\perp}^{\prime}\right) \not q_{1 \perp}}{d\left(k_{2}^{\prime}, k_{1}\right)}+\frac{x_{2}\left(x_{2} \not k_{\perp}^{\prime}-\not k_{2 \perp}\right) \not q_{1 \perp}-x_{1} \not q_{1 \perp}\left(x_{2} \not k_{\perp}^{\prime}-\not k_{2 \perp}\right)}{d\left(k_{2}, k_{1}^{\prime}\right)} \\
+\frac{q_{1 \perp}^{2}\left(x_{2}\left(\not k_{1 \perp}-x_{1} \not k_{\perp}^{\prime}\right) \not k_{\perp}^{\prime}-x_{1} \not k_{\perp}^{\prime}\left(\not k_{1 \perp}-x_{1} \not k_{\perp}^{\prime}\right)\right)}{k_{\perp}^{\prime 2} d\left(k_{2}^{\prime}, k_{1}\right)}+\frac{q_{1 \perp}^{2}\left(x_{1} \not k_{\perp}^{\prime}\left(x_{2} \not k_{\perp}^{\prime}-\not k_{2 \perp}\right)-x_{2}\left(x_{2} \not k_{\perp}^{\prime}-\not k_{2 \perp}\right) \not k_{\perp}^{\prime}\right)}{k_{\perp}^{\prime 2} d\left(k_{2}, k_{1}^{\prime}\right)} ; \\
\mathcal{L}_{2}^{a}=\frac{x_{2}\left(x_{2} \not k_{\perp}^{\prime}-\not k_{2 \perp}\right) \not q_{1 \perp}-x_{1} \not \not_{1 \perp}\left(x_{2} \not k_{\perp}^{\prime}-\not k_{2 \perp}\right)}{d\left(k_{2}, k_{1}^{\prime}\right)}-\frac{x_{1} \not q_{1 \perp}\left(\not k_{1 \perp}-x_{1} \not k_{\perp}^{\prime}\right)-x_{2}\left(\not k_{1 \perp}-x_{1} \not k_{\perp}^{\prime}\right) \phi_{1 \perp}}{d\left(k_{2}^{\prime}, k_{1}\right)} \\
-\frac{q_{1 \perp}^{2}\left(x_{2}\left(\not k_{1 \perp}-x_{1} \not k_{\perp}^{\prime}\right) \not k_{\perp}^{\prime}-x_{1} \not k_{\perp}^{\prime}\left(\not k_{1 \perp}-x_{1} \not k_{\perp}^{\prime}\right)\right)}{k_{\perp}^{\prime 2} d\left(k_{2}^{\prime}, k_{1}\right)}+\frac{q_{1 \perp}^{2}\left(x_{1} \not k_{\perp}^{\prime}\left(x_{2} \not k_{\perp}^{\prime}-\not k_{2}\right)-x_{2}\left(x_{2} \not k_{\perp}^{\prime}-\not k_{2 \perp}\right) \not k_{\perp}^{\prime}\right)}{k_{\perp}^{\prime 2} d\left(k_{2}, k_{1}^{\prime}\right)} \\
+2 \frac{x_{1} \not q_{1 \perp}\left(x_{2} \not k_{1 \perp}-x_{1} \not k_{2 \perp}\right)-x_{2}\left(x_{2} \not k_{1 \perp}-x_{1} \not k_{2 \perp}\right) \not q_{1 \perp}}{d\left(k_{2}, k_{1}\right)}
\end{gathered}
$$




$$
\begin{gathered}
+2 \frac{q_{1 \perp}^{2}}{k_{\perp}^{\prime 2} d\left(k_{2}, k_{1}\right)}\left(x_{2}\left(x_{2} \not k_{1 \perp}-x_{1} \not k_{2 \perp}\right) \not k_{\perp}^{\prime}-x_{1} \not k_{\perp}^{\prime}\left(x_{2} \not k_{1 \perp}-x_{1} \not k_{2 \perp}\right)\right) ; \\
\mathcal{L}_{3}^{a}=0 .
\end{gathered}
$$

In the case of $q \bar{q}$ production the particle $\tilde{P}$ in the sum (4.8) must be a quark with momentum $k_{1}^{\prime}$. Taking the representation (4.23), (4.28) for the vertex of quark-antiquark production in Reggeon-Reggeon collisions, (2.6) for the Quark-Quark-Reggeon vertex and summing over spin projections according to (4.30), we have:

$$
\mathcal{L}_{1}^{b}=-b\left(q_{1} ; k_{1}^{\prime}, k_{2}\right), \quad \mathcal{L}_{2}^{b}=-\frac{1}{2} \overline{b\left(q_{1} ; k_{2}, k_{1}^{\prime}\right)}, \quad \mathcal{L}_{3}^{b}=\frac{1}{2}\left(b\left(q_{1} ; k_{1}^{\prime}, k_{2}\right)-\overline{b\left(q_{1} ; k_{2}, k_{1}^{\prime}\right)}\right) .
$$

Quite analogously we obtain

$$
\mathcal{L}_{1}^{c}=-\overline{b\left(q_{1} ; k_{2}^{\prime}, k_{1}\right)}, \quad \mathcal{L}_{2}^{c}=\frac{1}{2} b\left(q_{1} ; k_{1}, k_{2}^{\prime}\right), \quad \mathcal{L}_{3}^{c}=-\frac{1}{2}\left(b\left(q_{1} ; k_{1}, k_{2}^{\prime}\right)-\overline{b\left(q_{1} ; k_{2}^{\prime}, k_{1}\right)}\right) .
$$

The functions $b\left(q_{1} ; k_{1}, k_{2}\right)$ and $\overline{b\left(q_{1} ; k_{2}, k_{1}\right)}$ are defined in (4.29).

The quantities $\mathcal{L}_{n}^{c}$ are easily obtained from (4.11) with account of the representation (4.23), (4.28) and are equal

$$
\begin{gathered}
\mathcal{L}_{1}^{d}=\frac{q_{1 \perp}^{2}}{k_{\perp}^{2}}\left(b\left(q_{1}-r ; k_{1}, k_{2}\right)-\overline{b\left(q_{1}-r ; k_{2}, k_{1}\right)}\right), \\
\mathcal{L}_{2}^{d}=-\frac{q_{1 \perp}^{2}}{k_{\perp}^{2}}\left(b\left(q_{1}-r ; k_{1}, k_{2}\right)+\overline{b\left(q_{1}-r ; k_{2}, k_{1}\right)}\right), \\
\mathcal{L}_{3}^{d}=0 .
\end{gathered}
$$

Since in the case of $q \bar{q}$ production the diargams Fig. $3 \mathrm{f}, \mathrm{g}$ can not contribute, Eqs. (4.33)(4.41) together with (4.18) determine the L.H.S. of the bootstrap equation (4.19). Using (4.23), (4.28) and (4.29) we can present the R.H.S. in the form

$$
g \frac{N_{c}}{2} \gamma_{c_{1} c_{2}}^{Q \bar{Q}}\left(q_{1}, q_{2}\right)=\frac{g^{3} N_{c}}{s \beta} \bar{u}\left(k_{1}\right) p_{2} \sum_{n=1}^{n=3} \mathcal{R}_{n}^{c_{1} c_{2}} \mathcal{L}_{n} v\left(k_{2}\right)
$$

where

$$
\begin{gathered}
\mathcal{L}_{1}=0 \\
\mathcal{L}_{2}=-\frac{1}{2}\left(b\left(q_{1} ; k_{1}, k_{2}\right)+\overline{b\left(q_{1} ; k_{2}, k_{1}\right)}\right) \\
\mathcal{L}_{3}=\frac{1}{2}\left(b\left(q_{1}-r ; k_{1}, k_{2}\right)-\overline{b\left(q_{1}-r ; k_{2}, k_{1}\right)}\right) .
\end{gathered}
$$




\section{Verification of the bootstrap equation}

We have to compare the coefficients in the decomposition into the colour structures $\mathcal{R}_{n}^{c_{1} c_{2}}$ in the left and right parts of the bootstrap equation (4.19). Let start with $\mathcal{R}_{1}^{c_{1} c_{2}}$. Consider sum of $\mathcal{L}_{1}^{i}$. Note that due to the symmetry of the integration measure in (4.19) under the substitution $r_{\perp} \rightarrow\left(q_{2 \perp}-r_{\perp}\right)$ we can make this substitution in separate terms in $\mathcal{L}_{1}^{i}$. Doing it in the terms with the denominator $D\left(k_{2}^{\prime}, k_{2}^{\prime}-q_{1}\right)$ permits to convert them in terms with the denominator $D\left(k_{1}^{\prime}-q_{1}, k_{1}^{\prime}\right)$. After that, using the decompositions

$$
\begin{aligned}
& \frac{x_{1} x_{2}}{d\left(k_{2}, k_{1}^{\prime}\right) D\left(k_{2}, k_{1}^{\prime}\right)}=\frac{1}{k_{\perp}^{\prime 2}}\left(\frac{1}{d\left(k_{2}, k_{1}^{\prime}\right)}-\frac{1}{D\left(k_{2}, k_{1}^{\prime}\right)}\right), \\
& \frac{x_{1} x_{2}}{d\left(k_{2}^{\prime}, k_{1}\right) D\left(k_{2}^{\prime}, k_{1}\right)}=\frac{1}{k_{\perp}^{\prime 2}}\left(\frac{1}{d\left(k_{2}^{\prime}, k_{1}\right)}-\frac{1}{D\left(k_{2}^{\prime}, k_{1}\right)}\right),
\end{aligned}
$$

it is easy to see, that the terms with the denominators

$$
D\left(k_{2}^{\prime}, k_{1}\right), \quad D\left(k_{2}, k_{1}^{\prime}\right), \quad D\left(k_{1}^{\prime}, k_{1}^{\prime}-q_{1}\right)
$$

are cancelled and we obtain for the sum of $\mathcal{L}_{1}^{i}$ :

$$
\frac{x_{1} x_{2} q_{1 \perp}^{2}}{d\left(k_{2}^{\prime}, k_{1}\right)}-\frac{x_{1} x_{2} q_{1 \perp}^{2}}{d\left(k_{2}, k_{1}^{\prime}\right)}+\frac{q_{1 \perp}^{2}\left(d\left(k_{2}^{\prime}, k_{1}\right)-x_{1} x_{2} k_{\perp}^{\prime 2}\right)}{k_{\perp}^{\prime 2} d\left(k_{2}^{\prime}, k_{1}\right)}+\frac{q_{1 \perp}^{2}\left(x_{1} x_{2} k_{\perp}^{\prime 2}-d\left(k_{2}, k_{1}^{\prime}\right)\right)}{k_{\perp}^{\prime 2} d\left(k_{2}, k_{1}^{\prime}\right)}=0
$$

as it must be, since the structure $\mathcal{R}_{1}^{c_{1} c_{2}}$ is absent in the R.H.S. of the bootstrap equation.

Turn to the colour structure $\mathcal{R}_{2}^{c_{1} c_{2}}$. Using (4.46), (4.47) we obtain from the sum of $\mathcal{L}_{2}^{i}$ :

$$
\begin{gathered}
-\frac{x_{1} x_{2} q_{1 \perp}^{2}}{d\left(k_{2}^{\prime}, k_{1}\right)}-\frac{x_{1} x_{2} q_{1 \perp}^{2}}{d\left(k_{2}, k_{1}^{\prime}\right)}+\frac{2}{d\left(k_{2}, k_{1}\right)}\left[2 x_{1} x_{2}\left(q_{1 \perp}\left(k_{1 \perp}+k_{2 \perp}\right)\right)-x_{1} \not q_{1 \perp} \not k_{2 \perp}-x_{2} \not k_{1 \perp} \not q_{1}\right] \\
+\frac{q_{1 \perp}^{2}}{k_{\perp}^{\prime 2} d\left(k_{2}^{\prime}, k_{1}\right)}\left(x_{1} x_{2} k_{\perp}^{\prime 2}-d\left(k_{2}^{\prime}, k_{1}\right)\right)+\frac{q_{1 \perp}^{2}}{k_{\perp}^{\prime 2} d\left(k_{2}, k_{1}^{\prime}\right)}\left(x_{1} x_{2} k_{\perp}^{\prime 2}-d\left(k_{2}, k_{1}^{\prime}\right)\right) \\
-\frac{q_{1 \perp}^{2}}{k_{\perp}^{\prime 2} d\left(k_{2}, k_{1}\right)}\left(2 x_{1} x_{2} k_{\perp}^{\prime 2}\right)+2 \frac{q_{1 \perp}^{2}}{k_{\perp}^{\prime 2}}+\frac{2 x_{1} x_{2} q_{1 \perp}^{2} \not k_{1 \perp} \not k_{2 \perp}}{d\left(k_{2}, k_{1}\right) D\left(k_{2}, k_{1}\right)} \\
-\frac{\left(\not k_{2 \perp}-\not q_{1 \perp}\right) \not k_{2 \perp}}{D\left(k_{2}, k_{2}-q_{1}\right)}-\frac{\not k_{1 \perp}\left(\not k_{1 \perp}-\not q_{1 \perp}\right)}{D\left(k_{1}-q_{1}, k_{1}\right)}+2 .
\end{gathered}
$$

One can readily see that the terms depending on $r_{\perp}$ cancel each other with the result:

$$
\begin{gathered}
-\frac{1}{D\left(k_{1}-q_{1}, k_{1}\right)}\left(\not k_{1 \perp}\left(\not k_{1 \perp}-\not q_{1 \perp}\right)\right)-\frac{1}{D\left(k_{2}, k_{2}-q_{1}\right)}\left(\left(\not k_{2 \perp}-\not q_{1 \perp}\right) \not k_{2} \perp\right) \\
-2 \frac{1}{d\left(k_{2}, k_{1}\right)}\left[x_{2} \not k_{1 \perp} \not q_{1 \perp}+x_{1} \not q_{1 \perp} \not k_{2 \perp}+x_{1} x_{2} q_{1 \perp}^{2}-2 x_{1} x_{2}\left(q_{1 \perp}\left(k_{1 \perp}+k_{2 \perp}\right)\right)\right] \\
+\frac{x_{1} x_{2}}{d\left(k_{2}, k_{1}\right) D\left(k_{2}, k_{1}\right)}\left(2 \not k_{1 \perp} \not k_{2 \perp} q_{1 \perp}^{2}\right)+2 .
\end{gathered}
$$

It is just $\mathcal{L}_{2}$, so that for the colour structure $\mathcal{R}_{3}^{c_{1} c_{2}}$ the bootstrap equation is satisfied. 
Al last, consider the colour structure $\mathcal{R}_{3}^{c_{1} c_{2}}$. For the sum of $\mathcal{L}_{3}^{i}$ we have

$$
\begin{aligned}
\frac{\not k_{1 \perp}^{\prime}\left(\not k_{1 \perp}^{\prime}-\not q_{1 \perp}\right)}{D\left(k_{1}^{\prime}-q_{1}, k_{1}^{\prime}\right)} & -\frac{\left(\not k_{2 \perp}-\not q_{1 \perp}\right) \not k_{2 \perp}}{D\left(k_{2}, k_{2}-q_{1}\right)}+\frac{\not k_{1 \perp}\left(\not k_{1 \perp}-\not q_{1 \perp}\right)}{D\left(k_{1}-q_{1}, k_{1}\right)}-\frac{\left(\not k_{2 \perp}^{\prime}-\not q_{1 \perp}\right) \not k_{2 \perp}^{\prime}}{D\left(k_{2}^{\prime}, k_{2}^{\prime}-q_{1}\right)} \\
& =\frac{\not k_{1 \perp}\left(\not k_{1 \perp}-\not \not_{1 \perp}\right)}{D\left(k_{1}-q_{1}, k_{1}\right)}-\frac{\left(\not k_{2 \perp}-\not q_{1 \perp}\right) \not k_{2 \perp}}{D\left(k_{2}, k_{2}-q_{1}\right)},
\end{aligned}
$$

that is exactly $\mathcal{L}_{3}$.

So, the bootstrap equation for $q \bar{q}$ production is satisfied.

\subsection{Two-gluon production}

\section{Denotations}

In the case of two-gluon production Eqs. (4.20)-(4.22) are applied as before; but now $k_{1}$ and $k_{2}$ are the gluon momenta. The effective vertex of two-gluon production in Reggeon-Reggeon collisions in a gauge invariant form was obtained in [34]. In the lightcone gauge (2.8) for both gluons the vertex takes the form:

$$
\begin{gathered}
\gamma_{i j}^{G_{1} G_{2}}\left(q_{1}, q_{2}\right)=4 g^{2}\left(e_{1 \perp}^{*}\right)_{\alpha}\left(e_{2 \perp}^{*}\right)_{\beta} \\
\times\left[\left(T^{i_{1}} T^{i_{2}}\right)_{i j} b^{\alpha \beta}\left(q_{1} ; k_{1}, k_{2}\right)+\left(T^{i_{2}} T^{i_{1}}\right)_{i j} b^{\beta \alpha}\left(q_{1} ; k_{2}, k_{1}\right)\right],
\end{gathered}
$$

where $e_{1,2}$ are the polarization vectors of the produced gluons, $i_{1,2}$ are their colour indices, $i, j$ are the colour indices of the Reggeons with momenta $q_{1}$ and $q_{2}$ correspondingly, and

$$
\begin{gathered}
b^{\alpha \beta}\left(q_{1} ; k_{1}, k_{2}\right)=\frac{1}{2} g_{\perp}^{\alpha \beta}\left[\frac{x_{1} x_{2}}{d\left(k_{2}, k_{1}\right)}\left(2 q_{1 \perp}\left(x_{1} k_{2}-x_{2} k_{1}\right)_{\perp}+q_{1 \perp}^{2}\left(x_{2}-\frac{x_{1} k_{2 \perp}^{2}}{D\left(k_{2}, k_{1}\right)}\right)\right)\right. \\
\left.-x_{2}\left(1-\frac{k_{1 \perp}^{2}}{D\left(q_{1}-k_{1}, k_{1}\right)}\right)\right]-\frac{x_{2} k_{1 \perp}^{\alpha} q_{1 \perp}^{\beta}-x_{1} q_{1 \perp}^{\alpha}\left(q_{1}-k_{1}\right)_{\perp}^{\beta}}{D\left(q_{1}-k_{1}, k_{1}\right)}-\frac{x_{1} q_{1 \perp}^{2} k_{1 \perp}^{\alpha}\left(q_{1}-k_{1}\right)^{\beta}}{k_{1 \perp}^{2} D\left(q_{1}-k_{1}, k_{1}\right)} \\
-\frac{x_{1} q_{1 \perp}^{\alpha}\left(x_{1} k_{2}-x_{2} k_{1}\right)_{\perp}^{\beta}+x_{2} q_{1 \perp}^{\beta}\left(x_{1} k_{2}-x_{2} k_{1}\right)_{\perp}^{\alpha}}{d\left(k_{2}, k_{1}\right)}+\frac{x_{1} q_{1 \perp}^{2} k_{1 \perp}^{\alpha} k_{2 \perp}^{\beta}}{k_{1 \perp}^{2} D\left(k_{2}, k_{1}\right)} \\
+\frac{x_{1} x_{2} q_{1 \perp}^{2}}{d\left(k_{2}, k_{1}\right) D\left(k_{2}, k_{1}\right)}\left[\left(x_{1} k_{2}-x_{2} k_{1}\right)_{\perp}^{\alpha} k_{2 \perp}^{\beta}+k_{1 \perp}^{\alpha}\left(x_{1} k_{2}-x_{2} k_{1}\right)_{\perp}^{\beta}\right] .
\end{gathered}
$$

Here we use the denotations (4.26). Note that one can come to (4.54) starting from the vertex in the gauge $e\left(k_{1}\right) p_{1}=0, e\left(k_{2}\right) p_{2}=0$ [35]. Our $b^{\alpha \beta}\left(q_{1} ; k_{1}, k_{2}\right)$ can be obtained from $c^{\alpha \beta}\left(k_{1}, k_{2}\right)$ defined in 35] be the gauge transformation

$$
b^{\alpha \beta}\left(q_{1} ; k_{1}, k_{2}\right)=\left(g_{\perp}^{\alpha \gamma}-2 \frac{k_{1 \perp}^{\alpha} k_{1 \perp}^{\gamma}}{k_{1 \perp}^{2}}\right) c_{\gamma}^{\beta}\left(k_{1}, k_{2}\right) .
$$




\section{Independent colour structures}

Contrary to the case of $q \bar{q}$ production where $\mathcal{F}_{i j}^{P_{1} P_{2}}\left(q_{1}, q_{2}, r\right)$ (4.18) has the most general form in colour space, here not all admitted colour structures are present. The number of all independent structures is readily calculated. Indeed, decomposition of product of two octets $\left(8 \otimes 8=1 \oplus 8_{s} \oplus 8_{a} \oplus 10 \oplus 10^{*} \oplus 27\right)$ contains 5 different irreducible representations, one of which enters two times. Such decomposition is valid for two Reggeons as well as for two gluons. Therefore, total number of admitted independent colour structures is 8 . It occurs that only three of them enter in $\mathcal{F}_{i j}^{G_{1} G_{2}}$. Actually it is predictable and is related to specific colour structures of the effective vertices for one-gluon (2.18) and two-gluon production (3.18),(4.53). These vertices are expressed in terms of the colour group generators in the adjoint representation. From properties of these generators it follows that only three independent tensors with four indices can be built from them. Of course, their choice is not unique. We accept the following:

$$
\mathcal{R}_{(1) i j}^{i_{1} i_{2}}=\frac{2}{N_{c}} \operatorname{Tr}\left(T^{i} T^{j} T^{i_{2}} T^{i_{1}}\right), \quad \mathcal{R}_{(2) i j}^{i_{1} i_{2}}=T_{i l}^{i_{1}} T_{l j}^{i_{2}}, \quad \mathcal{R}_{(3) i j}^{i_{1} i_{2}}=T_{i l}^{i_{2}} T_{l j}^{i_{1}} .
$$

It seems that our choice is the most appropriate, i.e. the coefficients with which these tensors enter in $\mathcal{F}_{i j}^{G_{1} G_{2}}$ are the least cumbersome.

Let us present each of the contributions $\mathcal{F}_{i j}^{m}$ entering in $\mathcal{F}_{i j}^{G_{1} G_{2}}\left(q_{1}, q_{2}, r\right)$ (4.18) in the form

$$
\mathcal{F}_{i j}^{m}=2 g^{3} N_{c} \sum_{n=1}^{n=3} \mathcal{R}_{(n) i j}^{i_{1} i_{2}}\left(e_{1 \perp}^{*}\right)_{\alpha}\left(e_{2 \perp}^{*}\right)_{\beta} \mathcal{L}_{m n}^{\alpha \beta} .
$$

Writing in the same form the right part of (4.19)

$$
g \frac{N_{c}}{2} \gamma_{i j}^{G_{1} G_{2}}\left(q_{1}, q_{2}\right)=2 g^{3} N_{c}\left(e_{1 \perp}^{*}\right)_{\alpha}\left(e_{2 \perp}^{*}\right)_{\beta} \sum_{n=1}^{n=3} \mathcal{R}_{(n) i j}^{i_{1} i_{2}} \mathcal{L}_{n}^{\alpha \beta}
$$

we have from (4.53)

$$
\mathcal{L}_{1}^{\alpha \beta}=0, \quad \mathcal{L}_{2}^{\alpha \beta}=b^{\alpha \beta}\left(q_{1} ; k_{1}, k_{2}\right), \quad \mathcal{L}_{3}^{\alpha \beta}=b^{\beta \alpha}\left(q_{1} ; k_{2}, k_{1}\right) .
$$

The coefficients $\mathcal{L}_{m n}^{\alpha \beta}$ in (4.57) are found by straightforward calculation using the vertices (2.9), (2.18), (3.18) and (4.53). With account of (4.18) the bootstrap condition (4.19) requires

$$
\int \frac{d^{D-2} r_{\perp}}{r_{\perp}^{2}\left(q_{2}-r\right)_{\perp}^{2}}\left(\mathcal{L}_{a n}^{\alpha \beta}+\mathcal{L}_{b n}^{\alpha \beta}+\mathcal{L}_{c n}^{\alpha \beta}+2 \mathcal{L}_{d n}^{\alpha \beta}+2 \mathcal{L}_{f n}^{\alpha \beta}\right)=\mathcal{L}_{n}^{\alpha \beta} \int \frac{d^{D-2} r_{\perp}}{r_{\perp}^{2}\left(q_{2}-r\right)_{\perp}^{2}}
$$

for each $n$.

\section{Verification of the bootstrap equation}

For $n=1$ we obtain:

$$
\mathcal{L}_{a 1}^{\alpha \beta}=g_{\perp}^{\alpha \beta} x_{1} x_{2}\left[\frac{\left(k_{1}-x_{1} k^{\prime}\right)_{\perp} Q_{\perp}}{d\left(k_{2}^{\prime}, k_{1}\right)}+\frac{\left(k_{2}-x_{2} k^{\prime}\right)_{\perp} Q_{\perp}}{d\left(k_{2}, k_{1}^{\prime}\right)}\right]
$$




$$
\begin{gathered}
-\frac{x_{1} Q_{\perp}^{\alpha}\left(k_{1}-x_{1} k^{\prime}\right)_{\perp}^{\beta}+x_{2}\left(k_{1}-x_{1} k^{\prime}\right)_{\perp}^{\alpha} Q_{\perp}^{\beta}}{\left(k_{1}-x_{1} k^{\prime}\right)_{\perp}^{2}}-\frac{x_{1} Q_{\perp}^{\alpha}\left(k_{2}-x_{2} k^{\prime}\right)_{\perp}^{\beta}+x_{2}\left(k_{2}-x_{2} k^{\prime}\right)_{\perp}^{\alpha} Q_{\perp}^{\beta}}{\left(k_{2}-x_{2} k^{\prime}\right)_{\perp}^{2}} \\
\mathcal{L}_{b 1}^{\alpha \beta}=-b^{\alpha \beta}\left(q_{1} ; k_{1}^{\prime}, k_{2}\right), \\
\mathcal{L}_{c 1}^{\alpha \beta}=-b^{\beta \alpha}\left(q_{1} ; k_{2}^{\prime}, k_{1}\right), \\
\mathcal{L}_{d 1}^{\alpha \beta}=\frac{q_{1 \perp}^{2}}{2 k_{\perp}^{\prime 2}}\left[b^{\alpha \beta}\left(q_{1}-r ; k_{1}, k_{2}\right)+b^{\beta \alpha}\left(q_{1}-r ; k_{2}, k_{1}\right)\right] \\
\mathcal{L}_{f 1}^{\alpha \beta}=-\frac{q_{1 \perp}^{2}}{2\left(q_{1}-k_{1}^{\prime}\right)_{\perp}^{2} k_{1 \perp}^{\prime 2}}\left(k_{1}^{\prime}-k_{1} \frac{k_{1 \perp}^{\prime 2}}{k_{1 \perp}^{2}}\right)_{\perp}^{\alpha}\left(q_{1}-k_{1}^{\prime}-k_{2} \frac{\left(q_{1}-k_{1}^{\prime}\right)_{\perp}^{2}}{k_{2 \perp}^{2}}\right)_{\perp}^{\beta} .
\end{gathered}
$$

Here and below $Q_{\perp}=\left(q_{1}-k^{\prime} q_{1 \perp}^{2} / k_{\perp}^{2}\right)_{\perp}$. According to (4.60) the integrated sum of $\mathcal{L}_{m 1}^{\alpha \beta}$ must be zero. One can track the cancellation of separate contributions using the decompositions (4.46), (4.47) and the change of variables $r_{\perp} \leftrightarrow\left(q_{2}-r\right)_{\perp}$, at which $D\left(q_{1}-k_{1}^{\prime}, k_{1}\right) \leftrightarrow D\left(k_{2}^{\prime}, q_{1}-\right.$ $k_{2}^{\prime}$ ) and consequently,

$$
\frac{-x_{1} q_{1 \perp}^{\alpha}\left(q_{1}-k_{1}^{\prime}\right)_{\perp}^{\beta}+x_{2} k_{1 \perp}^{\prime \alpha} q_{1}^{\beta}}{D\left(q_{1}-k_{1}^{\prime}, k_{1}^{\prime}\right)} \leftrightarrow \frac{-x_{1} k_{2 \perp}^{\prime \beta} q_{1 \perp}^{\alpha}+x_{2} q_{1 \perp}^{\beta}\left(q_{1}-k_{2}^{\prime}\right)_{\perp}^{\alpha}}{D\left(k_{2}^{\prime}, q_{1}-k_{2}^{\prime}\right)} .
$$

After that the cancellation of the terms with $g_{\perp}^{\alpha \beta}$ follows from trivial relations:

$$
\begin{aligned}
& 2 x_{1} k_{\perp}^{\prime}\left(k_{1}-x_{1} k^{\prime}\right)_{\perp}-k_{1 \perp}^{2}=-\left(d\left(k_{2}^{\prime}, k_{1}\right)+x_{1}^{2} k_{\perp}^{\prime 2}\right), \\
& 2 x_{2} k_{\perp}^{\prime}\left(k_{2}-x_{2} k^{\prime}\right)_{\perp}-k_{2 \perp}^{2}=-\left(d\left(k_{2}, k_{1}^{\prime}\right)+x_{2}^{2} k_{\perp}^{\prime 2}\right) .
\end{aligned}
$$

To see that the sum of all other terms is zero the equality

$$
\int \frac{d^{D-2} r_{\perp}}{r_{\perp}^{2}\left(q_{2}-r\right)_{\perp}^{2}}\left[x_{2}\left(1-\frac{k_{1 \perp}^{\prime 2}}{D\left(q_{1}-k_{1}^{\prime}, k_{1}^{\prime}\right)}\right)+x_{1}\left(1-\frac{k_{2}^{\prime 2}}{D\left(k_{2}^{\prime}, q_{1}-k_{2}^{\prime}\right)}\right)\right]=0
$$

which follows from the change of variables $r_{\perp} \leftrightarrow\left(q_{2}-r\right)_{\perp}$ and $x_{1}+x_{2}=1$, is helpful.

Let us turn to the case $n=2$ in (4.60). For separate terms in the integrand we obtain

$$
\begin{gathered}
\mathcal{L}_{a 2}^{\alpha \beta}=-x_{1} x_{2} g_{\perp}^{\alpha \beta}\left[\frac{Q_{\perp}\left(k_{2}-x_{2} k^{\prime}\right)_{\perp}}{d\left(k_{2}, k_{1}^{\prime}\right)}+\frac{Q_{\perp}\left(x_{2} k_{1}-x_{1} k_{2}\right)_{\perp}}{d\left(k_{2}, k_{1}\right)}\right] \\
+\frac{x_{1} Q_{\perp}^{\alpha}\left(x_{2} k_{1}-x_{1} k_{2}\right)_{\perp}^{\beta}+x_{2}\left(x_{2} k_{1}-x_{1} k_{2}\right)_{\perp}^{\alpha} Q_{\perp}^{\beta}}{d\left(k_{2}, k_{1}\right)}+\frac{x_{1} Q_{\perp}^{\alpha}\left(k_{2}-x_{2} k^{\prime}\right)_{\perp}^{\beta}+x_{2}\left(k_{2}-x_{2} k^{\prime}\right)_{\perp}^{\alpha} Q_{\perp}^{\beta}}{d\left(k_{2}, k_{1}^{\prime}\right)}
\end{gathered}
$$

$$
\mathcal{L}_{b 2}^{\alpha \beta}=b^{\alpha \beta}\left(q_{1} ; k_{1}^{\prime}, k_{2}\right)
$$




$$
\begin{gathered}
\mathcal{L}_{c 2}^{\alpha \beta}=b^{\beta \alpha}\left(q_{1} ; k_{2}^{\prime}, k_{1}\right)+b^{\alpha \beta}\left(q_{1} ; k_{1}, k_{2}^{\prime}\right), \\
\mathcal{L}_{d 2}^{\alpha \beta}=-\frac{q_{1 \perp}^{2}}{2 k_{\perp}^{\prime 2}} b^{\beta \alpha}\left(q_{1}-r ; k_{2}, k_{1}\right), \\
\mathcal{L}_{f 2}^{\alpha \beta}=\frac{q_{1 \perp}^{2}}{2\left(q_{1}-k_{1}^{\prime}\right)_{\perp}^{2} k_{1 \perp}^{\prime 2}}\left(k_{1}^{\prime}-k_{1} \frac{k_{1 \perp}^{\prime 2}}{k_{1 \perp}^{2}}\right)_{\perp}^{\alpha}\left(q_{1}-k_{1}^{\prime}-k_{2} \frac{\left(q_{1}-k_{1}^{\prime}\right)_{\perp}^{2}}{k_{2 \perp}^{2}}\right)_{\perp}^{\beta} .
\end{gathered}
$$

Although separate contributions in (4.60) are rather complicated, their sum can be greatly simplified using the equalities

$$
\begin{gathered}
\int \frac{d^{D-2} r_{\perp}}{r_{\perp}^{2}\left(q_{2}-r\right)_{\perp}^{2}}\left[\frac{x_{1} k_{1 \perp}^{\prime \alpha}\left(q_{1}-k_{1}^{\prime}\right)_{\perp}^{\beta}}{k_{1 \perp}^{\prime 2} D\left(q_{1}-k_{1}^{\prime}, k_{1}^{\prime}\right)}+\frac{x_{2} k_{2 \perp}^{\prime \beta}\left(q_{1}-k_{2}^{\prime}\right)_{\perp}^{\alpha}}{k_{2 \perp}^{\prime 2} D\left(k_{2}^{\prime}, q_{1}-k_{2}^{\prime}\right)}-\frac{k_{1 \perp}^{\prime \alpha}\left(q_{1}-k_{1}^{\prime}\right)_{\perp}^{\beta}}{k_{1 \perp}^{\prime 2}\left(q_{1}-k_{1}^{\prime}\right)_{\perp}^{2}}\right]=0, \\
\int \frac{d^{D-2} r_{\perp}}{r_{\perp}^{2}\left(q_{2}-r\right)_{\perp}^{2}}\left[\frac{x_{1} k_{1 \perp}^{\alpha} k_{2 \perp}^{\prime \beta}}{k_{1 \perp}^{2} D\left(k_{2}^{\prime}, k_{1}\right)}+\frac{x_{2} k_{1 \perp}^{\alpha} k_{2 \perp}^{\prime \beta}}{k_{2 \perp}^{\prime 2} D\left(k_{2}^{\prime}, k_{1}\right)}-\frac{k_{1 \perp}^{\alpha}\left(q_{1}-k_{1}^{\prime}\right)_{\perp}^{\beta}}{k_{1 \perp}^{2}\left(q_{1}-k_{1}^{\prime}\right)_{\perp}^{2}}\right]=0,
\end{gathered}
$$

which are readily follow from the change of variables $r_{\perp} \leftrightarrow\left(q_{2}-r\right)_{\perp}$, relations (4.67), (4.68) and not less trivial equality

$$
x_{2} k_{\perp}^{\prime \beta} k_{1 \perp}^{\prime \alpha}-x_{1} k_{2 \perp}^{\beta} k_{\perp}^{\prime \alpha}=k_{1 \perp}^{\prime \alpha}\left(x_{2} k_{1}^{\prime}-x_{1} k_{2}\right)_{\perp}^{\beta}+k_{2 \perp}^{\beta}\left(x_{2} k_{1}^{\prime}-x_{1} k_{2}\right)_{\perp}^{\alpha} .
$$

After that fulfillment of (4.60) for $n=2$ becomes plain.

Finally, consider (4.60) at $n=3$. For the coefficients $\mathcal{L}_{m 3}^{\alpha \beta}$ we obtain:

$$
\begin{gathered}
\mathcal{L}_{a 3}^{\alpha \beta}=x_{1} x_{2} g_{\perp}^{\alpha \beta}\left[\frac{Q_{\perp}\left(k_{2}-x_{2} k^{\prime}\right)_{\perp}}{d\left(k_{2}, k_{1}^{\prime}\right)}+\frac{Q_{\perp}\left(x_{2} k_{1}-x_{1} k_{2}\right)_{\perp}}{d\left(k_{2}, k_{1}\right)}\right] \\
-\frac{x_{1} Q_{\perp}^{\alpha}\left(k_{2}-x_{2} k^{\prime}\right)_{\perp}^{\beta}+x_{2} Q_{\perp}^{\beta}\left(k_{2}-x_{2} k^{\prime}\right)_{\perp}^{\alpha}}{d\left(k_{2}, k_{1}^{\prime}\right)}-\frac{x_{1} Q_{\perp}^{\alpha}\left(x_{2} k_{1}-x_{1} k_{2}\right)_{\perp}^{\beta}+x_{2} Q_{\perp}^{\beta}\left(x_{2} k_{1}-x_{1} k_{2}\right)_{\perp}^{\alpha}}{d\left(k_{2}, k_{1}\right)} \\
\mathcal{L}_{b 3}^{\alpha \beta}=b^{\beta \alpha}\left(q_{1} ; k_{2}, k_{1}^{\prime}\right) \\
\mathcal{L}_{c 3}^{\alpha \beta}=0 \\
\mathcal{L}_{d 3}^{\alpha \beta}=\frac{q_{1 \perp}^{2}}{2 k_{\perp}^{\prime 2}} b^{\beta \alpha}\left(q_{1}-r ; k_{2}, k_{1}\right) \\
\mathcal{L}_{f 3}^{\alpha \beta}=0
\end{gathered}
$$

Verification of (4.60) is rather simple here; the trivial equality

$$
2 x_{1} k_{\perp}^{\prime}\left(k_{2}-x_{2} k^{\prime}\right)_{\perp}+k_{1 \perp}^{\prime 2}=d\left(k_{2}, k_{1}^{\prime}\right)+x_{1}^{2} k_{\perp}^{\prime 2} .
$$

is helpful to perform it. 


\section{Summary and discussion}

In this paper we have calculated in the one-loop approximation the leading logarithmic corrections to the QCD amplitudes in the QMRK. We have considered two essentially different kinematics. In one of them two particles with limited invariant mass are produced in the fragmentation region of one of colliding particles. In another there are two gaps between rapidities of the produced particles and rapidities of colliding ones (production in the central region). The radiative corrections were calculated using the $s$-channel unitarity. In both cases we have found that the radiative corrections are just the same which are prescribed by the Reggeized form of the amplitudes. It is worth-while to note that this form of corrections appears as a result of miraculous cancellations between various contributions. The $s$-channel unitarity method used by us for the calculation is very economic. Using this method we have to consider only a few contributions, whereas number of Feynman diagrams is estimated by hundreds. Nevertheless, even in this approach the cancellations are quite impressive.

Since in the $s$-channel unitarity method the radiative corrections are expressed in terms of the Reggeon vertices, the cancellation appears as a result of fulfillment of Eqs. (3.11) and (4.19). Therefore these equations are the bootstrap conditions, necessary for compatibility of the Reggeized form of the amplitudes with the $s$-channel unitarity.

The gluon Reggeization is one of remarkable properties of QCD, very important at high energies. It is proved in the LLA, but still remains a hypothesis in the NLA. This hypothesis can be checked, and, hopefully, proved [24] using the bootstrap requirement, i.e. the demand of compatibility of the Reggeized form of the amplitudes with the $s$-channel unitarity. The requirement leads to an infinite set of the bootstrap relations for the scattering amplitudes. Fulfillment of these relations guarantees the Reggeized form of the radiative corrections order by order in perturbation theory. It occurs that all these relations can be satisfied if the Reggeon vertices and the gluon Regge trajectory submit to several bootstrap conditions. The proof of the gluon Reggeization in the LLA [9] is just demonstration that fulfilment of the bootstrap conditions in the leading order is sufficient to satisfy all bootstrap relations. Hopefully, the same can be done in the NLA [24]. There are no doubts that the Reggeized form of the QMRK amplitudes can be proved in such way. Since these amplitudes contain the gluon Regge trajectory and the Reggeon-Reggeon-gluon vertex in the leading order, the only new (compared with the LLA) thing which is required to perform the proof is fulfillment of the bootstrap conditions (3.11) and (4.19). We'll return to this question elsewhere.

Acknowledgments: One of us (V.S.F.) thanks the Alexander von Humboldt foundation for the research award, the Universität Hamburg and DESY for their warm hospitality while a part of this work was done.

\section{References}

[1] M. Gell-Mann, M.L. Goldberger, F.E. Low, E. Marx and F. Zachariasen, Phys. Rev. 133 B (1964) 145. 
[2] S. Mandelstam, Phys. Rev. 137 B (1964) 949.

[3] M.T. Grisaru, H.J. Schnitzer and H.-S. Tsao, Phys. Rev. Lett. 30 (1973) 811; Phys. Rev. D 8 (1973) 4498.

[4] L.N. Lipatov, Yad. Fiz. 23 (1976) 642 [Sov. J. Nucl. Phys. 23 (1976) 338].

[5] V.S. Fadin, E.A. Kuraev and L.N. Lipatov, Phys. Lett. B 60 (1975) 50; E.A. Kuraev, L.N. Lipatov and V.S. Fadin, Zh. Eksp. Teor. Fiz. 71 (1976) 840 [Sov. Phys. JETP 44 (1976) 443]; 72 (1977) 377 [45 (1977) 199]; Ya.Ya. Balitskii and L.N. Lipatov, Sov. J. Nucl. Phys. 28 (1978) 822.

[6] V.S. Fadin and V.E. Sherman, Pis'ma Zh. Eksp. Teor. Fiz. 23 (1976) 599 [Sov. Phys. JETP Lett.23 (1976) 548]; Zh. Eksp. Teor. Fiz. 72 (1977) 1640 [Sov. Phys. JETP 45 (1977) 861]; V. S. Fadin and R. Fiore, Phys. Rev. D 64 (2001) 114012 arXiv:hep-ph/0107010.

[7] A. V. Bogdan, V. Del Duca, V. S. Fadin and E. W. Glover, JHEP 0203 (2002) 032 arXiv:hep-ph/0201240.

[8] M. I. Kotsky, L. N. Lipatov, A. Principe and M. I. Vyazovsky, arXiv:hep-ph/0207169.

[9] Ya.Ya. Balitskii, L.N. Lipatov and V.S. Fadin, in Proceedings of Leningrad Winter School on Physics of Elementary Particles, Leningrad, 1979, 109 (in Russian).

[10] V.S. Fadin and L.N. Lipatov, Phys. Lett. B429 (1998) 127.

[11] M. Ciafaloni and G. Camici, Phys. Lett. B430 (1998) 349.

[12] V.S. Fadin and R. Fiore, Phys. Lett. B440 (1998) 359.

[13] V.S. Fadin, R. Fiore and A. Papa, Phys. Rev. D60 (1999) 074025.

[14] V.S. Fadin and D.A. Gorbachev, Pis'ma v Zh. Eksp. Teor. Fiz. 71 (2000) 322 [JETP Letters 71 (2000) 222]; Phys. Atom. Nucl. 63 (2000) 2157 [Yad. Fiz. 63 (2000) 2253].

[15] V.S. Fadin, R. Fiore, M.I. Kotsky and A. Papa, Phys. Rev. D61 (2000) 094005.

[16] V.S. Fadin, R. Fiore, M.I. Kotsky and A. Papa, Phys. Rev. D61 (2000) 094006.

[17] V.S. Fadin and A.D. Martin, Phys. Rev. D60 (1999) 114008.

[18] V. Fadin, D. Ivanov and M. Kotsky, In: New Trends in High-Energy Physics, Ed. L.L. Jenkovszky, Kiev, 2000, pp. 190-194, hep-ph/0007119.

[19] J. Bartels, S. Gieseke and C.F. Qiao, Phys. Rev. D63 (2001) 056014, hep-ph/0009102.

[20] V. Fadin, D. Ivanov, M. Kotsky, hep-ph/0106099.

[21] J. Bartels, S. Gieseke, A. Kyrieleis, Phys. Rev. D65 (2000) 014006, hep-ph/0107152.

[22] V.S. Fadin, R. Fiore and M.I. Kotsky, Phys. Lett. B494 (2000) 100, hep-ph/0007312. 
[23] V.S. Fadin, R. Fiore and A. Papa, Phys. Rev. D63 (2001) 034001, hep-ph/0008006.

[24] V.S. Fadin, Talk given at the NATO Advanced Research Workshop DIFFRACTION 2002, August 31 - September 6, 2002, Alushta, Crimea, Ukraine; to be published in the Proceedings.

[25] M. Braun, hep-ph/9901447.

[26] M. Braun and G.P. Vacca, Phys. Lett. B447 (2000) 156.

[27] V.S. Fadin, R. Fiore, M.I. Kotsky and A. Papa, Phys. Lett. B495 (2000) 329, hep-ph/0008057.

[28] M. Braun and G.P. Vacca, Phys. Lett. B454 (1999) 319.

[29] V. S. Fadin and A. Papa, Nucl. Phys. B 640 (2002) 309 arXiv:hep-ph/0206079

[30] V.S. Fadin and L.N. Lipatov, Nucl. Phys. B 406 (1993) 259.

[31] J. Bartels, Nucl. Phys. B 175 (1980) 365.

[32] V.S. Fadin and L.N. Lipatov, Nucl. Phys. B477 (1996) 767.

[33] V. S. Fadin, R. Fiore, A. Flachi and M. I. Kotsky, Phys. Lett. B 422 (1998) 287 arXiv:hep-ph/9711427; V. S. Fadin, M. I. Kotsky, R. Fiore and A. Flachi, Phys. Atom. Nucl. 62 (1999) 999 [Yad. Fiz. 62 (1999) 1066].

[34] L.N. Lipatov and V.S. Fadin, Zh. Eksp. Teor. Fiz. Pis'ma 49 (1989) 311 [Sov. Phys. JETP Lett. 49 (1989) 352]; Yad. Fiz. 50, (1989) 1141 [Sov. J. Nucl. Phys. 50 (1989) $712]$.

[35] V.S. Fadin, M.A. Kotsky and L.N. Lipatov, Phys. Lett. B 415 (1997) 97; Yad. Fiz. 61(6) (1998) 716. 